\title{
Determination of $\chi$ from liquid-liquid phase data in ternary polymer systems (solvent/polymer/polymer) with hydrogen bonding
}

\author{
J. E. Figueruelo', R. García-Lopera ${ }^{*}$, I. S. Monzó ${ }^{1}$, C. Abad², A. Campos ${ }^{1}$ \\ ${ }^{1}$ ICMUV, Departament de Química Física, Universitat de Valencia, 46100 Burjassot, Valencia, Spain \\ ${ }^{2}$ Departament de Bioquímica i Biología Molecular, Universitat de Valencia, 46100 Burjassot, Valencia, Spain
}

Received 19 December 2007; accepted in revised form 23 February 2008

\begin{abstract}
Two different models accounting for the dependences of $\chi$ and $g$ interaction parameters on both temperature and composition have been applied to different ternary polymer systems (TPS) solvent(1)/polymer(2)/polymer(3). The analyzed TPS have consisted on ten different polymer mixtures in chloroform as common solvent that can specifically interact via hydrogen bond. Experimental ternary phase diagrams determined by liquid chromatography were taken from literature. The application of the two models to the experimental data have served to obtain $\chi_{i j}$ and $g_{i j}$ interaction parameters for all the binary $i j(i j=12,13,23)$ interactions established between the three components of the system, with simplified mathematical procedures. The results have shown a fair agreement between the calculated and the literature values, at least when the model containing an empirical entropy correction is used. Moreover, the evaluated interaction parameters follow the expected behaviour as the content of the H-donor polymer in the mixture is raised and are also in good accordance with experimental viscometric data.
\end{abstract}

Keywords: modeling and simulation, interaction parameters, ternary polymer systems, hydrogen-bonding, rigid lattice theory

\section{Introduction}

An increasing interest in solving complex systems, such as polyblends [1], nanoparticles, composites or ternary polymer systems (TPS) formed by a mixture of three polymers, through the thermodynamic analysis of phase diagrams is clearly evidenced. For example, the curing of an epoxy in the presence of thermoplastics and additives is usually followed by the evolution of their phase diagrams as the curing process goes on. The classical procedure consists on writing the Gibbs free energy function of mixture and obtaining the corresponding chemical potentials, $\Delta \mu$, in each phase. Generally, the equations obtained for this kind of systems show a great complexity, and, in consequence, the values of the $\chi$ interaction parameters are usually unknown or rarely known with any certainty. To circumvent this drawback, different approximations have to be introduced in order to simplify the mathematical solution.

It is now generally accepted that in order to obtain quantitative agreement between Flory-Huggins theory and experimental observations for most polymer systems, $\chi$ interaction parameter dependence on both temperature and composition must be considered [2-4]. Sometimes the $g$ interaction parameter (mathematically related to $\chi$ ) can be expressed as a more complex function than $1 / T$ [5]. Other $g(T$, composition) functions can be theoretically derived in the framework of the rigid lattice model, as Staverman [6] and Koningsveld and Kleitjens [7], among others, have demonstrated. Nowadays it is

*Corresponding author, e-mail: rosa.garcia@uv.es

(C) BME-PT and GTE 
accepted that the dependence of $g$ on concentration mainly arises from the disparities in size and shape of the solvent molecules and the polymer segments. According to Staverman [6], it is possible to write the functionality of $g$ as Equation (1):

$$
g=\frac{D(T)}{1-c \phi_{2}}
$$

where $c$ is a constant determined from Bondi studies [8-10] and $D(T)$ accounts for the temperature dependence in the form of Equation (2):

$$
D(T)=a+\frac{b}{T}
$$

On the other hand, $\chi \rightarrow g$, just when the volume fraction of component $2 \phi_{2} \rightarrow 1$, or at infinite dilution of solvent, which are the conditions fulfilled by the inverse gas chromatography technique [5], although, it is also used (Equation (3)) when analyzing the thermodynamics of the phase separation during polymerization of a thermoset system into a thermoplastic matrix $[11,12]$.

$\chi=\frac{D(T)}{1-c \phi_{2}}$

Occasionally, and in order to adjust the experimental results, the interaction function, dependent on both temperature and composition, can be written $[5,13-15]$ as Equation (4):

$$
g=\alpha+\frac{D(T)}{1-\phi_{2}}
$$

where $\alpha$ is an empirical entropy correction [5] given by Equation (5):

$$
\alpha=\alpha_{S}+\frac{\alpha_{H}}{T}
$$

Obviously, this new $g$ value yields a different expression for $\chi$, (Equation (6)) [15]:

$$
\chi=\alpha+\frac{D(1-c)}{\left(1-c \phi_{2}\right)^{2}}
$$

Moreover, an even more complex function to express the $\chi$ temperature and concentration dependence has been found (Equation (7)) [16]:

$$
\chi\left(T, \phi_{2}\right)=\left(1+c_{1} \phi_{2}+c_{2} \phi_{2}^{2}\right)(D+d \ln T)
$$

where $c_{i}(i=1,2), D$ and $d$ being adjustable parameters.

Usually, the use of any of the above expressions simplifies the $\chi$ evaluation, although unfortunately, the calculated values cannot be experimentally checked, especially in complex systems. Fortunately, there are more traditional and simple systems with well-known values of the interaction parameters that can be used to judge the validity of the approximations used in the more complex and actual systems. In this regard, in recent papers, dealing with compatibility between polystyrene copolymers and other polymers in solution via hydrogen bonding [17-19], the phase diagrams of the ternary polymer systems (TPS) have been obtained from experimental liquid chromatography data [20-22]. The achievement of $\chi$ values from liquid-liquid phase data has been considered a reliable method [16] and consists in solving the equations obtained by equating the chemical potentials in both phases in equilibrium, for all the components. To mathematically solve the equations, two of the most often used models for $\chi\left(T, \phi_{2}\right)$ have been applied. The validity of the theoretical results has been judged in the light of the experimental ones, measured by viscometry and liquid chromatography [18-20]. Moreover, the adequacy and reliability of the diverse $\chi$ models has also been tested from a basic thermodynamic viewpoint.

\section{Theory}

\subsection{Chemical potentials and interaction parameters}

In ternary solvent(1)/polymer(2)/polymer(3) systems with polydisperse polymers, the Gibbs free energy change upon mixing $n_{i}$ mols of component $i$ $(i=1,2,3)$ is given by Equation (8):

$$
\begin{aligned}
\frac{\Delta G^{M}}{R T}= & n_{1} \ln \phi_{1}+\sum_{n=1, m_{2}}^{m_{2}} n_{2, x_{n}} \ln \phi_{2, x_{n}}+ \\
& \sum_{n=1, m_{3}}^{m_{3}} n_{3, x_{n}} \ln \phi_{3, x_{n}}+n_{1} \phi_{2} g_{12}+n_{1} \phi_{3} g_{13}
\end{aligned}
$$

whereas for monodisperse polymers (or with low polydispersity index) is given by Equation (9): 


$$
\frac{\Delta G^{M}}{R T}=n_{1} \ln \phi_{1}+n_{2} \ln \phi_{2}+n_{3} \ln \phi_{3}+n_{1} \phi_{2} g_{12}+n_{1} \phi_{3} g_{13}+\bar{x}_{2} n_{2} \phi_{3} g_{23}
$$

and the chemical potentials deduced from Equation (9) are then given by Equations (10) and (11):

$$
\begin{aligned}
\frac{\Delta \mu_{1}}{R T}= & \frac{1}{R T}\left(\frac{\partial \Delta G^{M}}{\partial n_{1}}\right)_{n_{2}, n_{3}, p, T}=\ln \phi_{1}+\left(1-\frac{V_{1}}{V_{2}}\right) \phi_{2}+\left(1-\frac{V_{1}}{V_{3}}\right) \phi_{3}+\left[g_{12}-\frac{\phi_{1} \phi_{2}}{1-\phi_{1}} \frac{\mathrm{d} g_{12}}{\mathrm{~d} \phi_{2}}\right] \phi_{2}\left(1-\phi_{1}\right)+ \\
& {\left[g_{13}-\frac{\phi_{1} \phi_{3}}{1-\phi_{1}} \frac{\mathrm{d} g_{13}}{\mathrm{~d} \phi_{3}}\right] \phi_{3}\left(1-\phi_{1}\right)-\left[g_{23}+\phi_{3} \frac{\mathrm{d} g_{23}}{\mathrm{~d} \phi_{3}}\right] \phi_{2} \phi_{3} \frac{V_{1}}{V_{2}}=} \\
& \ln \phi_{1}+\left(1-\frac{V_{1}}{V_{2}}\right) \phi_{2}+\left(1-\frac{V_{1}}{V_{3}}\right) \phi_{3}+\chi_{12} \phi_{2}\left(1-\phi_{1}\right)+\chi_{13} \phi_{3}\left(1-\phi_{1}\right)-\varepsilon_{23} \phi_{2} \phi_{3} \frac{V_{1}}{V_{2}} \\
\frac{\Delta \mu_{2}}{R T}= & \frac{1}{R T}\left(\frac{\partial \Delta G^{M}}{\partial n_{2}}\right)_{n_{1}, n_{3}, p, T}=\ln \phi_{2}+\left(1-\frac{V_{2}}{V_{1}}\right) \phi_{1}+\left(1-\frac{V_{2}}{V_{3}}\right) \phi_{3}+\left[g_{12}+\phi_{2} \frac{\mathrm{d} g_{12}}{\mathrm{~d} \phi_{2}}\right] \phi_{1}\left(1-\phi_{2}\right) \frac{V_{2}}{V_{1}}- \\
& {\left[g_{13}+\phi_{3} \frac{\mathrm{d} g_{13}}{\mathrm{~d} \phi_{3}}\right] \phi_{1} \phi_{3} \frac{V_{2}}{V_{1}}+\left[g_{23}-\frac{\phi_{2} \phi_{3}}{1-\phi_{2}} \frac{\mathrm{d} g_{23}}{\mathrm{~d} \phi_{3}}\right] \phi_{3}\left(1-\phi_{2}\right) \frac{V_{2}}{V_{1}}=} \\
& \ln \phi_{2}+\left(1-\frac{V_{2}}{V_{1}}\right) \phi_{1}+\left(1-\frac{V_{2}}{V_{3}}\right) \phi_{3}+\varepsilon_{12} \phi_{1}\left(1-\phi_{2}\right) \frac{V_{2}}{V_{1}}-\varepsilon_{13} \phi_{1} \phi_{3} \frac{V_{2}}{V_{1}}+\chi_{23} \phi_{3}\left(1-\phi_{2}\right) \frac{V_{2}}{V_{1}}
\end{aligned}
$$

and by Equation (12):

$$
\begin{aligned}
\frac{\Delta \mu_{3}}{R T}= & \frac{1}{R T}\left(\frac{\partial \Delta G^{M}}{\partial n_{3}}\right)_{n_{1}, n_{2}, p, T}=\ln \phi_{3}+\left(1-\frac{V_{3}}{V_{1}}\right) \phi_{1}+\left(1-\frac{V_{3}}{V_{2}}\right) \phi_{2}-\left[g_{12}+\phi_{2} \frac{\mathrm{d} g_{12}}{\mathrm{~d} \phi_{2}}\right] \phi_{1} \phi_{2} \frac{V_{3}}{V_{1}}+ \\
& {\left[g_{13}+\phi_{3} \frac{\mathrm{d} g_{13}}{\mathrm{~d} \phi_{3}}\right] \phi_{1}\left(1-\phi_{3}\right) \frac{V_{3}}{V_{1}}+\left[g_{23}+\phi_{3} \frac{\mathrm{d} g_{23}}{\mathrm{~d} \phi_{3}}\right] \phi_{2}\left(1-\phi_{3}\right) \frac{V_{3}}{V_{1}}=} \\
& \ln \phi_{3}+\left(1-\frac{V_{3}}{V_{1}}\right) \phi_{1}+\left(1-\frac{V_{3}}{V_{2}}\right) \phi_{2}-\varepsilon_{12} \phi_{1} \phi_{2} \frac{V_{3}}{V_{1}}+\varepsilon_{13} \phi_{1}\left(1-\phi_{3}\right) \frac{V_{3}}{V_{1}}+\varepsilon_{23} \phi_{2}\left(1-\phi_{3}\right) \frac{V_{3}}{V_{1}}
\end{aligned}
$$

where $n_{i}, V_{i}$ and $\Delta \mu_{i}(i=1,2,3)$ are mol numbers, molar volumes and chemical potentials, respectively. In Equations (10)-(12) the residual contributions to $\Delta \mu_{i}$ (the terms containing $g_{12}, g_{13}$ and $\left.g_{23}\right)$ are ternary volume fractions $\phi_{i}(i=1,2,3)$ functions representative of binary interaction parameters. Generally, two types of ternary functions can be considered, those of the form given in Equation (13):

$$
\left[g_{i j}-\frac{\phi_{i} \phi_{j}}{1-\phi_{i}} \frac{\mathrm{d} g_{i j}}{\mathrm{~d} \phi_{j}}\right]
$$

where $i j$ represents the 1-2, 1-3 and 2-3 interactions, which are also called $\chi_{i j}$ because when they are transformed into binary functions (concretely, $\chi_{13}^{\text {binary }}$ is deduced from $\chi_{13}^{\text {ternary }}$ by making $\phi_{2}=0$ ), the classical Flory's interaction parameters are obtained, which are defined in $\Delta \mu_{i}^{\text {binary }}$ by Equation (14) [15]:

$$
\chi_{i j}=g_{i j}-\phi_{i} \frac{\mathrm{d} g_{i j}}{\mathrm{~d} \phi_{j}}=g_{i j}+\phi_{i} \frac{\mathrm{d} g_{i j}}{\mathrm{~d} \phi_{i}}
$$

The other functions types adopt the following functionality shown by Equation (15): 


$$
g_{i j}+\phi_{j} \frac{\mathrm{d} g_{i j}}{\mathrm{~d} \phi_{j}}
$$

also named $\varepsilon_{i j}$, because when they are transformed into binary functions, the parameters defining $\Delta \mu_{i}^{\text {binary }}$ are obtained by Equation (16) $[15,23]$ :

$$
\varepsilon_{i j}=g_{i j}+\phi_{j} \frac{\mathrm{d} g_{i j}}{\mathrm{~d} \phi_{j}}=g_{i j}-\phi_{j} \frac{\mathrm{d} g_{i j}}{\mathrm{~d} \phi_{i}}
$$

The interconversion between $\chi_{i j}$ and $\varepsilon_{i j}$ for binary polymer systems (BPS) is achieved at constant temperature [24] through different dependences on component $j$ concentration, $\chi_{13}^{\text {binary }}$ expressed as volume fraction $\phi_{j}$ by Equations (17)-(20):

$$
\begin{aligned}
& \chi_{i j}=\chi_{i j}^{(0)}+\chi_{i j}^{(1)} \phi_{j}+\ldots=\sum_{n=0}^{m} \chi^{(n)} \phi_{j}^{n} \\
& \chi_{i j}=g_{i j}-\phi_{i} \frac{\mathrm{d} g_{i j}}{\mathrm{~d} \phi_{j}}=g_{i j}-(1-\phi j) \frac{\mathrm{d} g_{i j}}{\mathrm{~d} \phi_{j}} \\
& \chi_{i j}=-d\left[\left(1-\phi_{j}\right) g_{i j}\right] \\
& g_{i j}=\frac{1}{1-\phi_{j}} \int_{\phi_{j}}^{1} \chi_{i j} \mathrm{~d} \phi_{j}
\end{aligned}
$$

Finally, the substitution of Equation (20) into Equation (17) yields Equation (21):

$$
\begin{aligned}
g_{i j}= & \left(\chi_{i j}^{(0)}+\frac{\chi_{i j}^{(1)}}{2}+\ldots\right)+\left(\frac{\chi_{i j}^{(1)}}{2}+\frac{\chi_{i j}^{(2)}}{3}\right) \phi_{j}+\ldots= \\
& \sum_{n=0}^{m} \frac{\chi^{(n)}}{n+1} \frac{1-\phi_{j}^{(n+1)}}{1-\phi_{j}}
\end{aligned}
$$

which allows to evaluate $g_{i j}$ parameters from experimental $\chi_{i j}$ values.

\subsection{Models for interaction parameters dependences on concentration and temperature in BPS}

\subsubsection{Model a1}

In the literature is possible to find $g_{i j}$ values as a function of temperature and composition according to Equation (22) [5, 15]: $g_{i j}=\alpha_{i j}+\frac{D_{i j}}{1-c_{i j} \phi_{j}}$

where $\alpha_{i j}$ and $c_{i j}$ are constants for a given system and temperature interval, and $D_{i j}(T)$ (Equation (2)), being $a$ and $b$ parameters with entropic and enthalpic character, respectively [4]. According to Equation (18), the corresponding $\chi_{i j}$ values are given by Equation (23):

$\chi_{i j}=\alpha_{i j}+\frac{D_{i j}\left(1-c_{i j}\right)}{\left(1-c_{i j} \phi_{j}\right)^{2}}$

and according to Equation (16) the corresponding $\varepsilon_{i j}$ should be as given by Equation (24):

$\varepsilon_{i j}=\alpha_{i j}+\frac{D_{i j}}{\left(1-c_{i j} \phi_{j}\right)^{2}}$

There are other models describing the $g$ dependences with temperature and composition for binary polymer systems. In general it is accepted that the main reason for the $g$ dependence on concentration arises from the disparity in size and shape between the solvent molecules and the polymer segments. Therefore, and according to Staverman [6], the $g$ function can be written as given by Equation (25):

$$
g_{i j}=\frac{D_{i j}}{1-c_{i j} \phi_{j}}
$$

with $D_{i j}$ and $c_{i j}$ as previously defined. However, when this set of equations is applied to the experimental results, is necessary to introduce a new fitting empirical parameter, $\alpha_{i j}$. At high $\phi_{j}$ values, calculated and measured binodals, spinodals and $\chi_{i j}$ vs. $\phi_{j}$ curves show that the empirical $\alpha_{i j}$ parameter depends on temperature (Equation (26)):

$$
\alpha_{i j}=m+n T
$$

where the parameter $n$ may assume any sign and the relation can only describe the behaviour within a rather limited range of temperatures. As a consequence, it is arrived again at the Equation (22) with $\alpha_{i j}$ dependence with $T$. 


\subsubsection{Model a2}

Other models allow to express the $g_{i j}$ interaction parameters in BPS by using a less number of fitting parameters, according to Equation (27):

$$
g_{i j}=\frac{D_{i j}}{c_{i j}\left(1-\phi_{j}\right)} \ln \left(1-c_{i j} \phi_{j}\right)
$$

with $D_{i j}$ and $c_{i j}$ as defined in Equation (22). Now, the $\chi_{i j}$ values attained are given by Equation (28):

$\chi_{i j}=\frac{D_{i j}}{1-c_{i j} \phi_{j}}$

This expression has been recently used to thermodynamically analyze the phase separation occurring during the polymerization of a thermoset epoxy system into a thermoplastic matrix [11, 12, 25]. According to this model and taking into account Equation (27) the following $\varepsilon_{i j}$ value is obtained as shown by Equation (29):

$\varepsilon_{i j}=\frac{D_{i j}}{c_{i j}} \ln \left(1-c_{i j} \phi_{j}\right) \frac{\phi_{i}+\phi_{j}}{\phi_{i}^{2}}-\frac{\phi_{j}}{\phi_{i}} \frac{D_{i j}}{1-c_{i j} \phi_{j}}$

as well as by Equation (30):

$$
\begin{aligned}
\phi_{i}^{2} \varepsilon_{i j}= & \frac{D_{i j}}{c_{i j}} \ln \left(1-c_{i j} \phi_{j}\right)-\phi_{i} \phi_{j} \frac{D_{i j}}{1-c_{i j} \phi_{j}}= \\
& \int_{\phi_{j}}^{1} \chi_{i j} \mathrm{~d} \phi_{j}-\phi_{i} \phi_{j} \chi_{i j}
\end{aligned}
$$

which relates $\varepsilon_{i j}$ and $\chi_{i j}$ in binary polymeric systems $[11,12,25]$.

\subsection{Models for interaction parameters dependences on concentration and temperature in TPS}

The $g_{i j}$ values derived with the different models for BPS have no reasons to change when ternary solvent/polymer/polymer systems are considered. In fact, in the Gibbs free energy of mixing, $g_{i j}$ represents the energy interchanged by one contact $i-j$ (1-2, 1-3 and 2-3) independently of the presence of a third component (see Equation (8)). To prove this fact, next the corresponding expressions for the 1-2 interaction, as an example, will be derived.

\subsubsection{Model b1}

First of all and recalling the a1 model, the following expressions shown by Equations (31)-(34) hold in BPS:

$$
g_{12}=\alpha_{12}+\frac{D_{12}}{1-c_{12} \phi_{2}}
$$

$$
\frac{\mathrm{d} g_{12}}{\mathrm{~d} \phi_{2}}=\frac{c_{12} D_{12}}{\left(1-c_{12} \phi_{2}\right)^{2}}
$$

$\chi_{12}=g_{12}-\phi_{1} \frac{\mathrm{d} g_{12}}{\mathrm{~d} \phi_{2}}=\alpha_{12}+\frac{D_{12}\left(1-c_{12}\right)}{\left(1-c_{12} \phi_{2}\right)^{2}}$

$\varepsilon_{12}=g_{12}+\phi_{2} \frac{\mathrm{d} g_{12}}{\mathrm{~d} \phi_{2}}=\alpha_{12}+\frac{D_{12}}{\left(1-c_{12} \phi_{2}\right)^{2}}$

whereas for a TPS and recalling Equations (13), (31) and (32), Equation (33) yields Equation (35):

$\chi_{12}=g_{12}-\frac{\phi_{1} \phi_{2}}{1-\phi_{1}} \frac{\mathrm{d} g_{12}}{\mathrm{~d} \phi_{2}}$

or Equation (36):

$\chi_{12}=\alpha_{12}+\frac{D_{12}\left(1-\phi_{1}-c_{12} \phi_{2}\right)}{\left(1-\phi_{1}\right)\left(1-c_{12} \phi_{2}\right)^{2}}$

On the other hand, from Equations (16), (31) and (32) Equation (37) can be obtained:

$\varepsilon_{12}=g_{12}+\phi_{2} \frac{d g_{12}}{d \phi_{2}}=\alpha_{12}+\frac{D_{12}}{\left(1-c_{12} \phi_{2}\right)^{2}}$

which is the same expression as Equation (34) since $\varepsilon$ is identical both in BPS and in TPS, although by using their respective binary and ternary volume fractions, $\phi$, in the corresponding calculations.

In TPS with two phases ( $\alpha$ and $\beta$ ) present in equilibrium the following conditions, given by Equation (38) hold:

$$
\begin{aligned}
& \Delta \mu_{1}^{\alpha}=\Delta \mu_{1}^{\beta} \\
& \Delta \mu_{2}^{\alpha}=\Delta \mu_{2}^{\beta} \\
& \Delta \mu_{3}^{\alpha}=\Delta \mu_{3}^{\beta}
\end{aligned}
$$

that applied to the present model leads to Equations (39)-(41): 


$$
\begin{aligned}
& \ln \phi_{1}^{\alpha}+\left(1-\frac{V_{1}}{V_{2}}\right) \phi_{2}^{\alpha}+\left(1-\frac{V_{1}}{V_{3}}\right) \phi_{3}^{\alpha}+\left[\alpha_{12}+\frac{D_{12}\left(1-\phi_{1}^{\alpha}-c_{12} \phi_{2}^{\alpha}\right)}{\left(1-\phi_{1}^{\alpha}\right)\left(1-c_{12} \phi_{2}^{\alpha}\right)^{2}}\right] \phi_{2}^{\alpha}\left(1-\phi_{1}^{\alpha}\right)+ \\
& {\left[\alpha_{13}+\frac{D_{13}\left(1-\phi_{1}^{\alpha}-c_{13} \phi_{3}^{\alpha}\right)}{\left(1-\phi_{1}^{\alpha}\right)\left(1-c_{13} \phi_{3}^{\alpha}\right)^{2}}\right] \phi_{3}^{\alpha}\left(1-\phi_{1}^{\alpha}\right)-\left[\alpha_{23}+\frac{D_{23}}{\left(1-c_{23} \phi_{3}^{\alpha}\right)^{2}}\right] \frac{V_{1}}{V_{2}} \phi_{2}^{\alpha} \phi_{3}^{\alpha}=} \\
& \ln \phi_{1}^{\beta}+\left(1-\frac{V_{1}}{V_{2}}\right) \phi_{2}^{\beta}+\left(1-\frac{V_{1}}{V_{3}}\right) \phi_{3}^{\beta}+\left[\alpha_{12}+\frac{D_{12}\left(1-\phi_{1}^{\beta}-c_{12} \phi_{2}^{\beta}\right)}{\left(1-\phi_{1}^{\alpha}\right)\left(1-c_{12} \phi_{2}^{\beta}\right)^{2}}\right] \phi_{2}^{\beta}\left(1-\phi_{1}^{\beta}\right)+ \\
& {\left[\alpha_{13}+\frac{D_{13}\left(1-\phi_{1}^{\beta}-c_{13} \phi_{3}^{\beta}\right)}{\left(1-\phi_{1}^{\beta}\right)\left(1-c_{13} \phi_{3}^{\beta}\right)^{2}}\right] \phi_{3}^{\beta}\left(1-\phi_{1}^{\beta}\right)-\left[\alpha_{23}+\frac{D_{23}}{\left(1-c_{23} \phi_{3}^{\beta}\right)^{2}}\right] \frac{V_{1}}{V_{2}} \phi_{2}^{\beta} \phi_{3}^{\beta}} \\
& \ln \phi_{2}^{\alpha}+\left(1-\frac{V_{2}}{V_{1}}\right) \phi_{1}^{\alpha}+\left(1-\frac{V_{2}}{V_{3}}\right) \phi_{3}^{\alpha}+\left[\alpha_{12}+\frac{D_{12}}{\left(1-c_{12} \phi_{2}^{\alpha}\right)^{2}}\right] \phi_{1}^{\alpha}\left(1-\phi_{2}^{\alpha}\right) \frac{V_{2}}{V_{1}}- \\
& {\left[\alpha_{13}+\frac{D_{13}}{\left(1-c_{13} \phi_{3}^{\alpha}\right)^{2}}\right] \phi_{1}^{\alpha} \phi_{3}^{\alpha} \frac{V_{2}}{V_{1}}+\left[\alpha_{23}+\frac{D_{23}\left(1-\phi_{2}^{\alpha}-c_{23} \phi_{3}^{\alpha}\right)}{\left(1-\phi_{2}^{\alpha}\right)\left(1-c_{23} \phi_{3}^{\alpha}\right)^{2}}\right] \phi_{3}^{\alpha}\left(1-\phi_{2}^{\alpha}\right) \frac{V_{2}}{V_{1}}=} \\
& \ln \phi_{2}^{\beta}+\left(1-\frac{V_{2}}{V_{1}}\right) \phi_{1}^{\beta}+\left(1-\frac{V_{2}}{V_{3}}\right) \phi_{3}^{\beta}+\left[\alpha_{12}+\frac{D_{12}}{\left(1-c_{12} \phi_{2}^{\beta}\right)^{2}}\right] \phi_{1}^{\beta}\left(1-\phi_{2}^{\beta}\right) \frac{V_{2}}{V_{1}}- \\
& {\left[\alpha_{13}+\frac{D_{13}}{\left(1-c_{13} \phi_{3}^{\beta}\right)^{2}}\right] \phi_{1}^{\beta} \phi_{3}^{\beta} \frac{V_{2}}{V_{1}}+\left[\alpha_{23}+\frac{D_{23}\left(1-\phi_{2}^{\beta}-c_{23} \phi_{3}^{\beta}\right)}{\left(1-\phi_{2}^{\beta}\right)\left(1-c_{23} \phi_{3}^{\beta}\right)^{2}}\right] \phi_{3}^{\beta}\left(1-\phi_{2}^{\beta}\right) \frac{V_{2}}{V_{1}}} \\
& \ln \phi_{3}^{\alpha}+\left(1-\frac{V_{3}}{V_{1}}\right) \phi_{1}^{\alpha}+\left(1-\frac{V_{3}}{V_{2}}\right) \phi_{2}^{\alpha}-\left[\alpha_{12}+\frac{D_{12}}{\left(1-c_{12} \phi_{2}^{\alpha}\right)^{2}}\right] \phi_{1}^{\alpha} \phi_{2}^{\alpha} \frac{V_{3}}{V_{1}}+ \\
& {\left[\alpha_{13}+\frac{D_{13}}{\left(1-c_{13} \phi_{3}^{\alpha}\right)^{2}}\right] \phi_{1}^{\alpha}\left(1-\phi_{3}^{\alpha}\right) \frac{V_{3}}{V_{1}}+\left[\alpha_{23}+\frac{D_{23}}{\left(1-c_{23} \phi_{3}^{\alpha}\right)^{2}}\right] \phi_{2}^{\alpha}\left(1-\phi_{3}^{\alpha}\right) \frac{V_{3}}{V_{1}}=} \\
& \ln \phi_{3}^{\beta}+\left(1-\frac{V_{3}}{V_{1}}\right) \phi_{1}^{\beta}+\left(1-\frac{V_{3}}{V_{2}}\right) \phi_{2}^{\beta}-\left[\alpha_{12}+\frac{D_{12}}{\left(1-c_{12} \phi_{2}^{\beta}\right)^{2}}\right] \phi_{1}^{\beta} \phi_{2}^{\beta} \frac{V_{3}}{V_{1}}+ \\
& {\left[\alpha_{13}+\frac{D_{13}}{\left(1-c_{13} \phi_{3}^{\beta}\right)^{2}}\right] \phi_{1}^{\beta}\left(1-\phi_{3}^{\beta}\right) \frac{V_{3}}{V_{1}}+\left[\alpha_{23}+\frac{D_{23}}{\left(1-c_{23} \phi_{3}^{\beta}\right)^{2}}\right] \phi_{2}^{\beta}\left(1-\phi_{3}^{\beta}\right) \frac{V_{3}}{V_{1}}}
\end{aligned}
$$

The terms in brackets are the corresponding $\chi_{i j}$ and $\varepsilon_{i j}$ values $(i j=12,13,23)$ in phases $\alpha$ or $\beta$, according to the b1 model, that is, taking into account Equations (36) and (37).

\subsubsection{Model b2}

Similarly and according to the a 2 model, in PBS the generic Equations (27)-(29) should read for the 1-2 interaction as shown by Equations (42)-(44):

$$
g_{12}=\frac{D_{12}}{c_{12}\left(1-\phi_{2}\right)} \ln \left(1-c_{12} \phi_{2}\right)
$$

$$
\chi_{12}=\frac{D_{12}}{1-c_{12} \phi_{2}}
$$

$$
\varepsilon_{12}=\frac{D_{12} \ln \left(1-\phi_{2}\right)}{c_{12}} \frac{\phi_{1} \phi_{2}}{\phi_{1}^{2}}-\frac{\phi_{2}}{\phi_{1}} \frac{D_{12}}{1-c_{12} \phi_{2}}
$$


whereas for a TPS yields Equation (45):

$\chi_{12}=g_{12}-\frac{\phi_{1} \phi_{2}}{1-\phi_{1}} \frac{\mathrm{d} g_{12}}{\mathrm{~d} \phi_{2}}=\frac{D_{12}}{c_{12}} \frac{\ln \left(1-\phi_{2}\right)}{1-\phi_{2}}\left\{1-\frac{\phi_{1} \phi_{2}}{\left(1-\phi_{1}\right)\left(1-\phi_{2}\right)}\right\}+\frac{D_{12}}{1-c_{12} \phi_{2}} \frac{\phi_{1} \phi_{2}}{\left(1-\phi_{1}\right)\left(1-\phi_{2}\right)}$

and Equation (46):

$\varepsilon_{12}=g_{12}+\phi_{2} \frac{\mathrm{d} g_{12}}{\mathrm{~d} \phi_{2}}=\frac{D_{12}}{c_{12}} \frac{\ln \left(1-c_{12} \phi_{2}\right)}{\left(1-\phi_{2}\right)^{2}}-\frac{\phi_{2} D_{12}}{\left(1-\phi_{2}\right)\left(1-c_{12} \phi_{2}\right)}$

It is easy to prove that Equation (45) transforms into Equation (43) for BPS when $\phi_{3}=0$ and $\phi_{1} \phi_{2} /\left[\left(1-\phi_{1}\right)\left(1-\phi_{2}\right)\right]=1$; and Equation (46) into Equation (29) if $\phi_{3}=0$.
Finally, the substitution of $\chi_{12}$ and $\varepsilon_{12}$ values derived with the $\mathbf{b} \mathbf{2}$ model into Equations (10)-(12) when equilibrium condition holds (Equation (38)), yields for each component of the TPS Equations (47) and (48):

$$
\begin{aligned}
& \ln \phi_{1}^{\alpha}+\left(1-\frac{V_{1}}{V_{2}}\right) \phi_{2}^{\alpha}+\left(1-\frac{V_{1}}{V_{3}}\right) \phi_{3}^{\alpha}+ \\
& {\left[\frac{D_{12}}{c_{12}}+\frac{\ln \left(1-\phi_{2}^{\alpha}\right)}{1-\phi_{2}^{\alpha}}\left\{1-\frac{\phi_{1}^{\alpha} \phi_{2}^{\alpha}}{\left(1-\phi_{1}^{\alpha}\right)\left(1-\phi_{2}^{\alpha}\right)}\right\}+\frac{\phi_{1}^{\alpha} \phi_{2}^{\alpha}}{\left(1-\phi_{1}^{\alpha}\right)\left(1-\phi_{2}^{\alpha}\right)} \frac{D_{12}}{1-c_{12} \phi_{2}^{\alpha}}\right] \phi_{2}^{\alpha}\left(1-\phi_{1}^{\alpha}\right)+} \\
& {\left[\frac{D_{13}}{c_{13}}+\frac{\ln \left(1-\phi_{3}^{\alpha}\right)}{1-\phi_{3}^{\alpha}}\left\{1-\frac{\phi_{1}^{\alpha} \phi_{3}^{\alpha}}{\left(1-\phi_{1}^{\alpha}\right)\left(1-\phi_{3}^{\alpha}\right)}\right\}+\frac{\phi_{1}^{\alpha} \phi_{3}^{\alpha}}{\left(1-\phi_{1}^{\alpha}\right)\left(1-\phi_{3}^{\alpha}\right)} \frac{D_{13}}{1-c_{13} \phi_{3}^{\alpha}}\right] \phi_{3}^{\alpha}\left(1-\phi_{3}^{\alpha}\right)-} \\
& D_{13}\left[\frac{\ln \left(1-c_{23} \phi_{3}^{\alpha}\right)}{c_{23}\left(1-\phi_{3}^{\alpha}\right)^{2}}-\frac{\phi_{3}^{\alpha}}{\left(1-\phi_{3}^{\alpha}\right)\left(1-c_{23} \phi_{3}^{\alpha}\right)}\right] \frac{V_{1}}{V_{2}} \phi_{2}^{\alpha} \phi_{3}^{\alpha}= \\
& \ln \phi_{1}^{\beta}+\left(1-\frac{V_{1}}{V_{2}}\right) \phi_{2}^{\beta}+\left(1-\frac{V_{1}}{V_{3}}\right) \phi_{3}^{\beta}+ \\
& {\left[\frac{D_{12}}{c_{12}}+\frac{\ln \left(1-\phi_{2}^{\beta}\right)}{1-\phi_{2}^{\beta}}\left\{1-\frac{\phi_{1}^{\beta} \phi_{2}^{\beta}}{\left(1-\phi_{1}^{\beta}\right)\left(1-\phi_{2}^{\beta}\right)}\right\}+\frac{\phi_{1}^{\beta} \phi_{2}^{\beta}}{\left(1-\phi_{1}^{\beta}\right)\left(1-\phi_{2}^{\beta}\right)} \frac{D_{12}}{1-c_{12} \phi_{2}^{\beta}}\right] \phi_{2}^{\beta}\left(1-\phi_{1}^{\beta}\right)+} \\
& {\left[\frac{D_{13}}{c_{13}}+\frac{\ln \left(1-\phi_{3}^{\beta}\right)}{1-\phi_{3}^{\beta}}\left\{1-\frac{\phi_{1}^{\beta} \phi_{3}^{\beta}}{\left(1-\phi_{1}^{\beta}\right)\left(1-\phi_{3}^{\beta}\right)}\right\}+\frac{\phi_{1}^{\beta} \phi_{3}^{\beta}}{\left(1-\phi_{1}^{\beta}\right)\left(1-\phi_{3}^{\beta}\right)} \frac{D_{13}}{1-c_{13} \phi_{3}^{\beta}}\right] \phi_{3}^{\beta}\left(1-\phi_{3}^{\beta}\right)-} \\
& D_{23}\left[\frac{\ln \left(1-c_{23} \phi_{3}^{\beta}\right)}{c_{23}\left(1-\phi_{3}^{\beta}\right)^{2}}-\frac{\phi_{3}}{\left(1-\phi_{3}^{\beta}\right)\left(1-c_{23} \phi_{3}^{\alpha}\right)}\right] \frac{V_{1}^{\beta} \phi_{3}^{\beta}}{V_{2}}
\end{aligned}
$$




$$
\begin{aligned}
& \ln \phi_{2}^{\alpha}+\left(1-\frac{V_{2}}{V_{1}}\right) \phi_{1}^{\alpha}+\left(1-\frac{V_{2}}{V_{3}}\right) \phi_{3}^{\alpha}+ \\
& D_{12}\left[\frac{\ln \left(1-c_{12} \phi_{2}^{\alpha}\right)}{c_{12}\left(1-\phi_{2}^{\alpha}\right)^{2}}-\frac{\phi_{2}^{\alpha}}{\left(1-\phi_{2}^{\alpha}\right)\left(1-c_{12} \phi_{2}^{\alpha}\right)}\right] \phi_{1}^{\alpha}\left(1-\phi_{2}^{\alpha}\right) \frac{V_{2}}{V_{1}}-D_{13}\left[\frac{\ln \left(1-c_{13} \phi_{3}^{\alpha}\right)}{c_{13}\left(1-\phi_{3}^{\alpha}\right)^{2}}-\frac{\phi_{3}^{\alpha}}{\left(1-\phi_{3}^{\alpha}\right)\left(1-c_{13} \phi_{3}^{\alpha}\right)}\right] \phi_{1}^{\alpha} \phi_{3}^{\alpha} \frac{V_{2}}{V_{1}}+ \\
& {\left[\frac{D_{23}}{c_{23}} \frac{\ln \left(1-\phi_{3}^{\alpha}\right)}{1-\phi_{3}^{\alpha}}\left\{1-\frac{\phi_{2}^{\alpha} \phi_{3}^{\alpha}}{\left(1-\phi_{2}^{\alpha}\right)\left(1-\phi_{3}^{\alpha}\right)}\right\}+\frac{\phi_{3}^{\alpha} D_{23}}{\left(1-\phi_{2}^{\alpha}\right)\left(1-\phi_{3}^{\alpha}\right)\left(1-c_{13} \phi_{3}^{\alpha}\right)}\right] \phi_{3}^{\alpha}\left(1-\phi_{2}^{\alpha}\right) \frac{V_{2}}{V_{1}}=} \\
& \ln \phi_{2}^{\beta}+\left(1-\frac{V_{2}}{V_{1}}\right) \phi_{1}^{\beta}+\left(1-\frac{V_{2}}{V_{3}}\right) \phi_{3}^{\beta}+ \\
& D_{12}\left[\frac{\ln \left(1-c_{12} \phi_{2}^{\beta}\right)}{c_{12}\left(1-\phi_{2}^{\beta}\right)^{2}}-\frac{\phi_{2}^{\beta}}{\left(1-\phi_{2}^{\beta}\right)\left(1-c_{12} \phi_{2}^{\beta}\right)}\right] \phi_{1}^{\beta}\left(1-\phi_{2}^{\beta}\right) \frac{V_{2}}{V_{1}}-D_{13}\left[\frac{\ln \left(1-c_{13} \phi_{3}^{\beta}\right)}{c_{13}\left(1-\phi_{3}^{\beta}\right)^{2}}-\frac{\phi_{3}^{\beta}}{\left(1-\phi_{3}^{\beta}\right)\left(1-c_{13} \phi_{3}^{\beta}\right)}\right] \phi_{1}^{\beta} \phi_{3}^{\beta} \frac{V_{2}}{V_{1}}+ \\
& {\left[\frac{D_{23}^{\beta} \phi_{3}^{\beta} D_{23}}{c_{23}} \frac{\ln \left(1-\phi_{3}^{\beta}\right)}{1-\phi_{3}^{\beta}}\left\{1-\frac{\phi_{2}^{\beta}}{\left(1-\phi_{2}^{\beta}\right)\left(1-\phi_{3}^{\beta}\right)}\right\}+\frac{V_{2}^{\beta}}{\left(1-\phi_{2}^{\beta}\right)\left(1-\phi_{3}^{\beta}\right)\left(1-c_{13} \phi_{3}^{\beta}\right)} \phi_{3}^{\beta}\left(1-\phi_{2}^{\beta}\right) \frac{V_{2}}{V_{1}}\right.}
\end{aligned}
$$

and Equation (49):

$$
\begin{aligned}
& \ln \phi_{3}^{\alpha}+\left(1-\frac{V_{3}}{V_{1}}\right) \phi_{1}^{\alpha}+\left(1-\frac{V_{3}}{V_{2}}\right) \phi_{2}^{\alpha}-D_{12}\left[\frac{\ln \left(1-c_{12} \phi_{2}^{\alpha}\right)}{c_{12}\left(1-\phi_{2}^{\alpha}\right)^{2}}-\frac{\phi_{2}^{\alpha}}{\left(1-\phi_{2}^{\alpha}\right)\left(1-c_{12} \phi_{2}^{\alpha}\right)}\right] \phi_{1}^{\alpha} \phi_{2}^{\alpha} \frac{V_{3}}{V_{1}}+ \\
& D_{13}\left[\frac{\ln \left(1-c_{13} \phi_{3}^{\alpha}\right)}{c_{13}\left(1-\phi_{3}^{\alpha}\right)^{2}}-\frac{\phi_{3}^{\alpha}}{\left(1-\phi_{3}^{\alpha}\right)\left(1-c_{13} \phi_{3}^{\alpha}\right)}\right] \phi_{1}^{\alpha}\left(1-\phi_{3}^{\alpha}\right) \frac{V_{3}}{V_{1}}+D_{23}\left[\frac{\ln \left(1-c_{23} \phi_{3}^{\alpha}\right)}{c_{23}\left(1-\phi_{3}^{\alpha}\right)^{2}}-\frac{\phi_{3}^{\alpha}}{\left(1-\phi_{3}^{\alpha}\right)\left(1-c_{23} \phi_{3}^{\alpha}\right.}\right] \phi_{2}^{\alpha}\left(1-\phi_{3}^{\alpha}\right) \frac{V_{3}}{V_{1}}= \\
& \ln \phi_{3}^{\beta}+\left(1-\frac{V_{3}}{V_{1}}\right) \phi_{1}^{\beta}+\left(1-\frac{V_{3}}{V_{2}}\right) \phi_{2}^{\beta}-D_{12}\left[\frac{\ln \left(1-c_{12} \phi_{2}^{\beta}\right)}{c_{12}\left(1-\phi_{2}^{\beta}\right)^{2}}-\frac{\phi_{2}^{\beta}}{\left(1-\phi_{2}^{\beta}\right)\left(1-c_{12} \phi_{2}^{\beta}\right)}\right] \phi_{1}^{\beta} \phi_{2}^{\beta} \frac{V_{3}}{V_{1}}+ \\
& D_{13}\left[\frac{\ln \left(1-c_{13} \phi_{3}^{\beta}\right)}{c_{13}\left(1-\phi_{3}^{\beta}\right)^{2}}-\frac{\phi_{3}^{\beta}}{\left(1-\phi_{3}^{\beta}\right)\left(1-c_{13} \phi_{3}^{\beta}\right)}\right] \phi_{1}^{\beta}\left(1-\phi_{3}^{\beta}\right) \frac{V_{3}}{V_{1}}+D_{23}\left[\frac{\ln \left(1-c_{23} \phi_{3}^{\beta}\right)}{c_{23}\left(1-\phi_{3}^{\beta}\right)^{2}}-\frac{\phi_{3}^{\beta}}{\left(1-\phi_{3}^{\beta}\right)\left(1-c_{23} \phi_{3}^{\beta}\right.}\right] \phi_{2}^{\beta}\left(1-\phi_{3}^{\beta}\right) \frac{V_{3}}{V_{1}}
\end{aligned}
$$

Again, the terms in brackets correspond to the $\chi_{i j}$ and $\varepsilon_{i j}(i j=12,13,23)$ interaction parameters in phases $\alpha$ or $\beta$ derived with $\mathbf{b} 2$ model.
It is interesting to note that both b1 (Equation (39) or (40)) and b2 (Equation (47) or (48)) models lead to the following expressions shown by Equations (50) and (51) when $\phi_{3}=0$ and $\phi_{1}+\phi_{2}=1$, that is, in binary conditions:

$$
\begin{aligned}
& \ln \phi_{1}^{\alpha}+\left(1-\frac{V_{1}}{V_{2}}\right) \phi_{2}^{\alpha}+\chi_{12}^{\alpha}\left(\phi_{1}^{\alpha}\right)^{2}=\ln \phi_{1}^{\beta}+\left(1-\frac{V_{1}}{V_{2}}\right) \phi_{2}^{\beta}+\chi_{12}^{\beta}\left(\phi_{1}^{\beta}\right)^{2} \\
& \ln \phi_{2}^{\alpha}+\left(1-\frac{V_{2}}{V_{1}}\right) \phi_{1}^{\alpha}+\varepsilon_{12}^{\alpha}\left(\phi_{1}^{\alpha}\right)^{2} \frac{V_{2}}{V_{1}}=\ln \phi_{2}^{\beta}+\left(1-\frac{V_{2}}{V_{1}}\right) \phi_{1}^{\beta}+\varepsilon_{12}^{\beta}\left(\phi_{1}^{\beta}\right)^{2} \frac{V_{2}}{V_{1}}
\end{aligned}
$$


that are those obtained for BPS [23, 25], evidencing the reliability of the models in spite of the mathematical complexity.

The advantage of using $\chi(T, \phi)$ and $\varepsilon(T, \phi)$ from models is the important reduction of the number of unknowns when mathematically solving the Equations (10)-(12). Once equilibrium compositions of each component in both phases are known $\left(\phi_{i}^{\alpha}\right.$ and $\phi_{i}^{\beta}$ ), the system of equations has 12 unknowns to determine, namely: $g_{i j}^{\alpha}, g_{i j}^{\beta},\left(\mathrm{d} g_{i j} / \mathrm{d} \phi_{j}\right)^{2},(i j=12,13$, 23). However, if model b1 is applied, in Equations (39)-(41) remain only 9 unknowns: $\alpha_{i j}, D_{i j}$ and $c_{i j}(i j=12,13,23)$. The reduction is more drastic if model b2 is applied, since only 6 unknowns remain in the Equations (47)-(49): $D_{i j}$ and $c_{i j}$ $(i j=12,13,23)$. In order to solve such systems, an additional number of equations must be generated,
6 in the case of b1 model and 3 in the $\mathbf{b} 2$ one, as it will be explained in the following.

\section{Results and discussion}

First of all, let us describe the TPS for which the calculations have been focused. They are ten polymer(2)/polymer(3) mixtures in $\mathrm{CHCl}_{3}$ as solvent (component(1)), namely: polystyrene (PS)/poly (vinyl pyridine) (PVPy), poly(styrene-co-methacrylic acid) (PSMAA-3)/PVPy, PSMAA-6/PVPy, PSMAA-7/PVPy, poly(styrene-co-vinyl phenol) (PSVPh-2)/PVPy, PSVPh-4/PVPy, PSVPh-7/PVPy, PS/poly(vinyl pyrrolidone) (PVP), PSMAA-3/PVP and PSVPh-2/PVP; where the number following MMA or $\mathrm{VPh}$ indicates the percentage (\% in weight) of comonomer in the corresponding copoly-

Table 1. Experimental compositions in equilibrium, $\phi_{i}^{\gamma}(i=1,2 ; \gamma=\alpha, \beta)$, from the binodal curve and randomly generated, $u_{i}^{\gamma}$ and $v_{i}^{\gamma}$ through b1 model (Equations (39)-(41)), for different ternary solvent(1)/polymer(2)/polymer(3) systems

\begin{tabular}{|c|c|c|c|c|c|c|c|c|c|c|c|}
\hline$\phi_{1}^{\alpha}$ & $\phi_{2}^{\alpha}$ & $\phi_{1}^{\beta}$ & $\phi_{2}^{\beta}$ & $\mathbf{u}_{1}^{\alpha}$ & $\mathbf{u}_{2}^{\alpha}$ & $\mathbf{u}_{1}^{\beta}$ & $\mathbf{u}_{2}^{\beta}$ & $v_{1}^{\alpha}$ & $v_{2}^{\alpha}$ & $v_{1}^{\beta}$ & $v_{2}^{\beta}$ \\
\hline \multicolumn{12}{|c|}{$\mathrm{CHCl}_{3} / \mathrm{PS} / \mathrm{PVPy}$} \\
\hline 0.9607 & 0.0297 & 0.9753 & 0.0042 & 0.9630 & 0.0298 & 0.9733 & 0.0042 & 0.9606 & 0.0297 & 0.9708 & 0.0042 \\
\hline 0.9678 & 0.0194 & 0.9761 & 0.0074 & 0.9701 & 0.0194 & 0.9741 & 0.0074 & 0.9677 & 0.0194 & 0.9716 & 0.0074 \\
\hline 0.9559 & 0.0378 & 0.9781 & 0.0013 & 0.9582 & 0.0379 & 0.9761 & 0.0013 & 0.9558 & 0.0378 & 0.9736 & 0.0013 \\
\hline \multicolumn{12}{|c|}{$\mathrm{CHCl}_{3} / \mathrm{PSMMA-3/PVPy}$} \\
\hline 0.7993 & 0.1745 & 0.9523 & 0.0072 & 0.8012 & 0.1749 & 0.9503 & 0.0072 & 0.7992 & 0.1745 & 0.9479 & 0.0072 \\
\hline 0.8552 & 0.1182 & 0.96 & 0.0096 & 0.8573 & 0.1185 & 0.9580 & 0.0096 & 0.8551 & 0.1182 & 0.9556 & 0.0096 \\
\hline 0.9290 & 0.0486 & 0.9578 & 0.0181 & 0.9312 & 0.0487 & 0.9558 & 0.0181 & 0.9289 & 0.0486 & 0.9534 & 0.0180 \\
\hline \multicolumn{12}{|c|}{$\mathrm{CHCl}_{3} / \mathrm{PSMMA-6/PVPy}$} \\
\hline 0.7917 & 0.1734 & 0.9112 & 0.0204 & 0.7936 & 0.1738 & 0.9093 & 0.0204 & 0.7916 & 0.1734 & 0.9070 & 0.0203 \\
\hline 0.8240 & 0.1409 & 0.9078 & 0.0317 & 0.8260 & 0.1412 & 0.9059 & 0.0316 & 0.8239 & 0.1409 & 0.9036 & 0.0316 \\
\hline 0.8803 & 0.0723 & 0.8982 & 0.0482 & 0.8824 & 0.0725 & 0.8963 & 0.0481 & 0.8802 & 0.0723 & 0.8941 & 0.0480 \\
\hline \multicolumn{12}{|c|}{$\mathrm{CHCl}_{3} / \mathrm{PSMMA-7/PVPy}$} \\
\hline 0.7670 & 0.2087 & 0.9047 & 0.0097 & 0.7668 & 0.2092 & 0.9028 & 0.0097 & 0.7669 & 0.2087 & 0.9006 & 0.0097 \\
\hline 0.8680 & 0.0925 & 0.8995 & 0.0371 & 0.8701 & 0.0927 & 0.8976 & 0.0370 & 0.8679 & 0.0925 & 0.8954 & 0.0369 \\
\hline 0.9541 & 0.0241 & 0.9644 & 0.0134 & 0.9564 & 0.0242 & 0.9624 & 0.0134 & 0.9540 & 0.0241 & 0.9600 & 0.0133 \\
\hline \multicolumn{12}{|c|}{$\mathrm{CHCl}_{3} / \mathrm{PSVPh}-2 / \mathrm{PVPy}$} \\
\hline 0.9009 & 0.0843 & 0.9643 & 0.008 & 0.9031 & 0.0845 & 0.9623 & 0.0080 & 0.9008 & 0.0843 & 0.9599 & 0.0080 \\
\hline 0.9369 & 0.0463 & 0.9656 & 0.0109 & 0.9392 & 0.0464 & 0.9636 & 0.0109 & 0.9368 & 0.0463 & 0.9618 & 0.0109 \\
\hline 0.9541 & 0.0241 & 0.9644 & 0.0134 & 0.9564 & 0.0242 & 0.9624 & 0.0134 & 0.9540 & 0.0241 & 0.9600 & 0.0133 \\
\hline \multicolumn{12}{|c|}{$\mathrm{CHCl}_{3} / \mathrm{PSVPh}-4 / \mathrm{PVPy}$} \\
\hline 0.8833 & 0.0814 & 0.9443 & 0.0056 & 0.8854 & 0.0816 & 0.9423 & 0.0559 & 0.8832 & 0.0814 & 0.9400 & 0.0056 \\
\hline 0.9238 & 0.0445 & 0.9442 & 0.0161 & 0.9260 & 0.0446 & 0.9422 & 0.0161 & 0.9237 & 0.0445 & 0.9399 & 0.0160 \\
\hline \multicolumn{12}{|c|}{$\mathrm{CHCl}_{3} / \mathrm{PSVPh}-7 / \mathrm{PVPy}$} \\
\hline 0.9211 & 0.0646 & 0.9671 & 0.0051 & 0.9233 & 0.0648 & 0.9651 & 0.0051 & 0.9210 & 0.0646 & 0.9627 & 0.0051 \\
\hline 0.9397 & 0.0446 & 0.9629 & 0.0138 & 0.9420 & 0.0447 & 0.9609 & 0.0138 & 0.9396 & 0.0446 & 0.9585 & 0.0137 \\
\hline \multicolumn{12}{|c|}{$\mathrm{CHCl}_{3} / \mathrm{PS} / \mathrm{PVP}$} \\
\hline 0.9212 & 0.0675 & 0.9511 & 0.0155 & 0.9234 & 0.0677 & 0.9491 & 0.0155 & 0.9211 & 0.0675 & 0.9467 & 0.0154 \\
\hline 0.9349 & 0.0498 & 0.9522 & 0.0189 & 0.9372 & 0.0499 & 0.9502 & 0.0189 & 0.9348 & 0.0498 & 0.9478 & 0.0188 \\
\hline \multicolumn{12}{|c|}{$\mathrm{CHCl}_{3} / \mathrm{PSMMA}-3 / \mathrm{PVP}$} \\
\hline 0.8277 & 0.0891 & 0.8990 & 0.0024 & 0.8297 & 0.0893 & 0.8971 & 0.0024 & 0.8276 & 0.0891 & 0.8949 & 0.0024 \\
\hline 0.8741 & 0.0467 & 0.8904 & 0.0208 & 0.8735 & 0.0468 & 0.8885 & 0.0208 & 0.8713 & 0.0467 & 0.8863 & 0.0207 \\
\hline \multicolumn{12}{|c|}{$\mathrm{CHCl}_{3} / \mathrm{PSVPh}-2 / \mathrm{PVP}$} \\
\hline 0.8370 & 0.0927 & 0.9016 & 0.0067 & 0.8390 & 0.0929 & 0.8997 & 0.0067 & 0.8369 & 0.0927 & 0.8975 & 0.0067 \\
\hline 0.8781 & 0.0447 & 0.8985 & 0.0169 & 0.8802 & 0.0448 & 0.8966 & 0.0169 & 0.8780 & 0.0447 & 0.8944 & 0.0168 \\
\hline
\end{tabular}


mers. The supplier, characterization and physicochemical properties of all the chemicals used have been recently described, as well as the experimental ternary phase diagrams determined by liquid chromatography with SEC columns [17]. These diagrams have served to obtain all the phase compositions for each component through the tie-lines joining the two phases in equilibrium at the binodal or cloud-point isotherm as explained elsewhere [17, 20-22].

Next, following the methods and procedure described in the Theory section, the volume fractions $\phi_{i}^{\gamma}(i=1,2,3 ; \gamma=\alpha, \beta)$ have been determined for all the phases equilibria of the above TPS and are compiled in Table 1. As mentioned above, Equations (39)-(41) generated through the model b1 contain 9 unknowns: $\alpha_{i j}, D_{i j}$ and $c_{i j}(i j=12,13$, 23) and in order to solve the system, 6 additional equations should be written. These additional equations are analogous in form to Equations (39)-(41) but with new compositions randomly generated, that differ from the original ones by a quantity no larger than $\pm 0.5 \%$. To do this, the original compositions in phase $\alpha\left(\phi_{i}^{\alpha}\right)$ are multiplied by the function $\left(1+0.005\right.$ Random) [26] and those in phase $\beta\left(\phi_{i}^{\beta}\right)$ by (1-0.005 Random). Therefore, a new set of compositions, namely $u_{i}^{\gamma}(i=1,2,3 ; \gamma=\alpha, \beta)$ are obtained. The third set, $v_{i}^{\gamma}(i=1,2,3 ; \gamma=\alpha, \beta)$, is generated by multiplying the original compositions by the function (1-0.005 Random). Summarizing, the 9 equations are: the (39)-(41) ones formed with $\phi_{i}^{\gamma}, 3$ with $u_{i}^{\gamma}$ (compositions very close to $\phi_{i}^{\gamma}$ and with unknowns concentration dependent assumed to remain invariants) and 3 more with $v_{i}^{\gamma}$ (also very close to $\phi_{i}^{\gamma}$ with unknowns also invariant). It must be remarked that $u_{i}^{\gamma}$ and $v_{i}^{\gamma}$ are randomly generated,

Table 2. Interaction parameters evaluated from Equations (39)-(41) for different ternary polymer systems

\begin{tabular}{|c|c|c|c|c|c|c|c|c|c|c|c|c|c|c|}
\hline$\phi_{1}^{\alpha}$ & $\chi_{12}^{\alpha}$ & $\chi_{12}^{\beta}$ & $\chi_{13}^{\alpha}$ & $\chi_{13}^{\beta}$ & $\chi_{23}^{\alpha}$ & $\chi_{23}^{\beta}$ & $\varepsilon_{23}^{\alpha}$ & $\varepsilon_{23}^{\beta}$ & $\mathbf{g}_{12}^{\alpha}$ & $\mathbf{g}_{12}^{\beta}$ & $\mathbf{g}_{13}^{\alpha}$ & $\mathbf{g}_{13}^{\beta}$ & $\mathbf{g}_{23}^{\alpha}$ & $\mathbf{g}_{23}^{\beta}$ \\
\hline \multicolumn{15}{|c|}{$\mathrm{CHCl}_{3} / \mathrm{PS} / \mathrm{PVPy}$} \\
\hline .9607 & 319 & 5870 & 533 & 5418 & 0.1204 & 1559 & 0.1526 & 0.2150 & 5020 & 5045 & 0.5607 & 5612 & 1551 & .2153 \\
\hline 0.9678 & 760 & 5560 & 5350 & 5810 & 0.0043 & 0.0035 & 0.0003 & 0.0005 & 0.5760 & 0.5770 & 0.4530 & 0.4520 & 0.0003 & 0.0005 \\
\hline 0.9559 & 545 & 5000 & 322 & 5667 & 0.0870 & 0.0930 & 0.0887 & 0.1086 & 5540 & .5550 & 5260 & 0.5250 & 0.0900 & 0.1022 \\
\hline 0.9597 & 4965 & 4737 & 5350 & 5470 & 0.0162 & 0.1395 & 0.0480 & 0.4340 & 0.4630 & 0.4670 & 0.5640 & 0.5650 & 0.0490 & 0.4407 \\
\hline 0.9645 & 570 & 6400 & 1650 & 0.5801 & 0120 & .0120 & 0.0120 & 0.0120 & 6440 & 6420 & 0.4020 & 0.3980 & .0120 & 0.0120 \\
\hline \multicolumn{15}{|c|}{$\mathrm{CHCl}_{3} / \mathrm{PSMMA}-3 / \mathrm{PVPy}$} \\
\hline 7993 & 5500 & 6910 & 2240 & 0.6330 & 0.0130 & 0.0130 & 0.0110 & 0.0120 & 1.1200 & 0.7520 & 0.1310 & 0.1160 & 0.0110 & 0.0120 \\
\hline 0.8552 & 5390 & 6530 & 4970 & 0.5220 & .0830 & 0.0740 & 0.0430 & -0.0335 & 1.0805 & 0.7665 & 0.0970 & 0.0920 & -0.0440 & -0.0341 \\
\hline 290 & 120 & 6690 & 3560 & 5340 & .0410 & 0.0420 & -0.0510 & -0.0520 & 9090 & 6910 & 1380 & 0.9534 & 0.0520 & -0.0530 \\
\hline \multicolumn{15}{|c|}{$\mathrm{CHCl}_{3} / \mathrm{PSMMA-6/PVPy}$} \\
\hline 0.7917 & 5630 & 8430 & 0.2860 & 0.4950 & 0.0810 & 0.0530 & 0.0520 & 0.0044 & $\mid 1.0610$ & 0.9230 & 0.2320 & 0.2060 & 0.0520 & 0.0041 \\
\hline 0.8240 & 740 & 5730 & 5800 & .6110 & .0780 & .0670 & 0.0630 & 0.0330 & .5720 & 5720 & 0.5650 & 0.5610 & -0.0640 & -0.0340 \\
\hline 8803 & 800 & 6980 & 5670 & 0.6400 & .0041 & -0.0041 & -0.0045 & -0.0047 & 0.8113 & 0.8010 & 0.2670 & 0.2600 & -0.0074 & -0.0048 \\
\hline \multicolumn{15}{|c|}{$\mathrm{CHCl}_{3} / \mathrm{PSMMA}-7 / \mathrm{PVPy}$} \\
\hline 7670 & 5800 & 6890 & 0.4310 & 0.4370 & 0.1280 & -0.1720 & -0.1440 & -0.2140 & $\mid 0.7000$ & 0.6910 & 0.4500 & 0.4500 & -0.1500 & -0.2230 \\
\hline 680 & 650 & 6540 & 820 & 6010 & .0390 & .0310 & -0.0270 & -0.0002 & 700 & 700 & 0 & 4220 & -0.0280 & -0.0002 \\
\hline \multicolumn{15}{|c|}{$\mathrm{CHCl}_{3} / \mathrm{PSVPh}-2 / \mathrm{PVPy}$} \\
\hline 0.9009 & 5410 & 5700 & 7751 & 0.7722 & 0.1160 & 0.0221 & -0.0680 & -0.0690 & 0.4901 & 0.5003 & .8242 & 0.8244 & -0.0690 & -0.0690 \\
\hline 0.9369 & 6010 & 0.6692 & 4041 & 0.5253 & 0.0191 & 0.0173 & -0.0142 & -0.0086 & 0.7392 & 0.7261 & 0.3111 & 0.3074 & -0.0140 & -0.0087 \\
\hline .9541 & 5441 & 0.6683 & 4490 & 0.4970 & .0044 & .0043 & -0.0022 & -0.0022 & 0.7480 & 0.7440 & 0.2960 & 0.2931 & -0.0022 & -0.0021 \\
\hline \multicolumn{15}{|c|}{ CHCl3/PSVPh-4/PVPy } \\
\hline 9238 & 7550 & 0.7582 & 5140 & 0.5981 & .1280 & 0.2318 & -0.5170 & -0.6800 & 0.7620 & 0.7611 & 0.5932 & 0.5881 & -0.5260 & -0.6911 \\
\hline \multicolumn{15}{|c|}{ CHCl3/PSVPh-7/PVPy } \\
\hline 0.9211 & 5820 & 0.5670 & 5050 & 0.5402 & -0.2291 & -0.2493 & -0.2603 & -0.2653 & 0.5590 & 0.5622 & 0.4651 & 0.4643 & -0.2641 & -0.2702 \\
\hline 0.9397 & 5840 & 0.6192 & 4571 & 0.5403 & -0.0370 & -0.0391 & -0.0392 & -0.0423 & 0.6661 & 0.6592 & 0.3772 & 0.3743 & -0.0402 & -0.0420 \\
\hline \multicolumn{15}{|c|}{$\mathrm{CHCl3/PS/PVP}$} \\
\hline 0.9212 & 5921 & 0.6961 & 0.5903 & 0.5900 & 0.3391 & 0.2762 & 0.2991 & 0.1830 & 0.7862 & 0.7623 & 0.5881 & 0.5882 & 0.3042 & 0.1850 \\
\hline 0.9349 & 6470 & 0.6581 & 0.5542 & 0.5271 & 0.3072 & 0.3051 & 0.3011 & 0.2912 & 0.6760 & 0.6742 & 0.5703 & 0.5724 & 0.3052 & 0.2851 \\
\hline \multicolumn{15}{|c|}{ CHCl3/PSMMA-3/PVP } \\
\hline 0.8277 & 7001 & 0.6362 & 4741 & 0.5603 & .1771 & 0.1652 & -0.1461 & -0.0290 & 0.6342 & 0.6333 & 0.3920 & 0.3853 & -0.1481 & -0.0291 \\
\hline 0.8714 & 7382 & 0.7753 & 0.4480 & 0.4751 & -0.0007 & -0.0003 & 0.0027 & 0.0033 & 0.8461 & 0.8311 & 0.3250 & 0.3233 & 0.0028 & 0.0033 \\
\hline \multicolumn{15}{|c|}{ CHCl3/PSVPh-2/PVP } \\
\hline 0.8370 & 7270 & 0.7510 & 0.3490 & 0.3951 & .0172 & .0101 & 0.0015 & 0.0152 & 0.9370 & 0.8752 & 0.3060 & 0.3021 & 0.0015 & 0.0154 \\
\hline 0.8781 & .7641 & 0.7893 & 0.4622 & 0.4971 & -0.0072 & -0.0076 & -0.0103 & -0.0107 & 0.8261 & 0.8172 & 0.2953 & 0.2931 & -0.0103 & -0.0108 \\
\hline
\end{tabular}


therefore their equations should not be lineal combinations of those formed with $\phi_{i}^{\gamma}$. Both composition sets, $u_{i}^{\gamma}$ and $v_{i}^{\gamma}$, have been also included in Table 1. Note that the values corresponding to component 3 have been omitted given by Equation (52):

$$
\sum_{i=1}^{3} \phi_{i}=\sum_{i=1}^{3} u_{i}=\sum_{i=1}^{3} v_{i}
$$

Once solved the 9 equations system, the $\alpha_{i j}, D_{i j}$ and $c_{i j}(i j=12,13,23)$ values are obtained being then possible to evaluate the 14 interaction parameters for each compositions set. In Table 2 are gathered the obtained results with b1 model for all the studied systems.

Let us now discuss the values of the interaction parameters, starting by those determined with the model b1 (listed in Table 2). The analysis will be done on the $\chi_{i j}^{\gamma}(i j=12,13,23 ; \gamma=\alpha, \beta)$ and $\varepsilon_{i j}^{\alpha}$ values since they are better known than the $g_{i j}^{\gamma}$ parameters [24, 27-29]. In Figure 1, the dependence of the binary $\chi_{12}^{\alpha}$ and $\chi_{12}^{\beta}$ parameters with the binary compositions $\phi_{2}^{\beta}=\phi_{2} /\left(1-\phi_{3}\right)$ is plotted for the system $\mathrm{CHCl}_{3} / \mathrm{PS} / \mathrm{PVPy}$, as an example. For the sake of comparison, Figure 1 also shows some $\chi_{12}$ values of $\mathrm{CHCl}_{3} / \mathrm{PS}$ from the literature [30]. The observed behaviour is the typical trend for good solvents and it seems that no disagreement takes place between experimental and theoretical values, neither with the values $\chi_{12}^{0}=0.51,0.53$ [24] or with $g_{12}^{0}=0.543,0.720$ [24], where the superscript 0 means infinite dilution. Similar $\chi_{13}^{\alpha}$ and $\chi_{13}^{\beta}$ values (Table 2) are found at any composition (a narrow composition range), just slightly larger than those

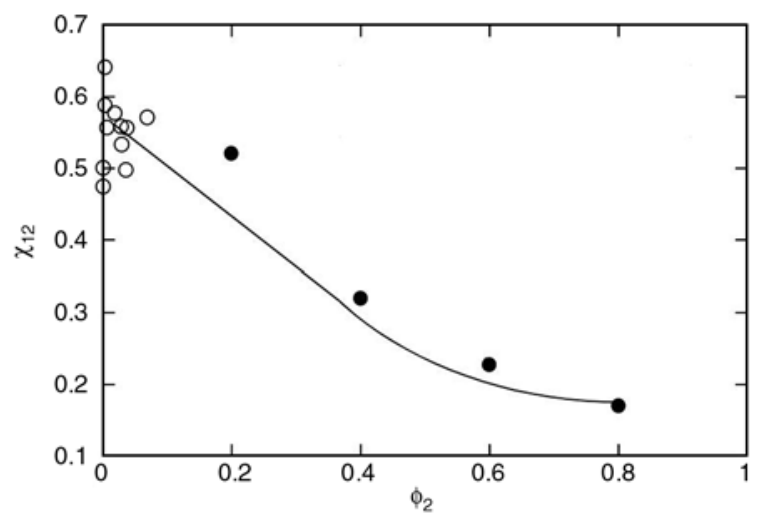

Figure 1. Dependence with composition of $\chi_{12}$ interaction parameters evaluated in this paper (0) and literature ones $(\bullet)[30]$, for the $\mathrm{CHCl}_{3}(1) / \mathrm{PS}(2)$ system found in the literature: $\chi_{13}=0.35,0.44$ [24]. Regarding the $\chi_{23}$ and $\varepsilon_{23}$ values, they appear always lower than experimental, as expected [14, 19].

The $\chi_{i j}^{\gamma}$ and $\varepsilon_{i j}^{\gamma}$ values for the systems $\mathrm{CHCl}_{3} /$ PSMAA-x/PVPy with three different MAA contents are also gathered in Table 2, showing similar behaviours to those above discussed with pure PS as component 2 but with some differences which deserve to be commented. Since no appreciable changes in the values of $\chi_{12}^{\alpha}, \chi_{12}^{\beta}, \chi_{13}^{\alpha}, \ldots$ at the diverse compositions have been found, it should be more illustrative to use mean values for the parameters; which are listed in Table 3. As seen, $\chi_{12}^{\alpha}$ and $\chi_{12}^{\beta}$ values smoothly increase with the MAA content in the copolymer, in agreement with the behaviour followed by their intrinsic viscosities, $\left[\eta_{2}\right]$ [17], shown in Figure 2a. The $\chi_{13}^{\alpha}$ and $\chi_{13}^{\beta}$ values

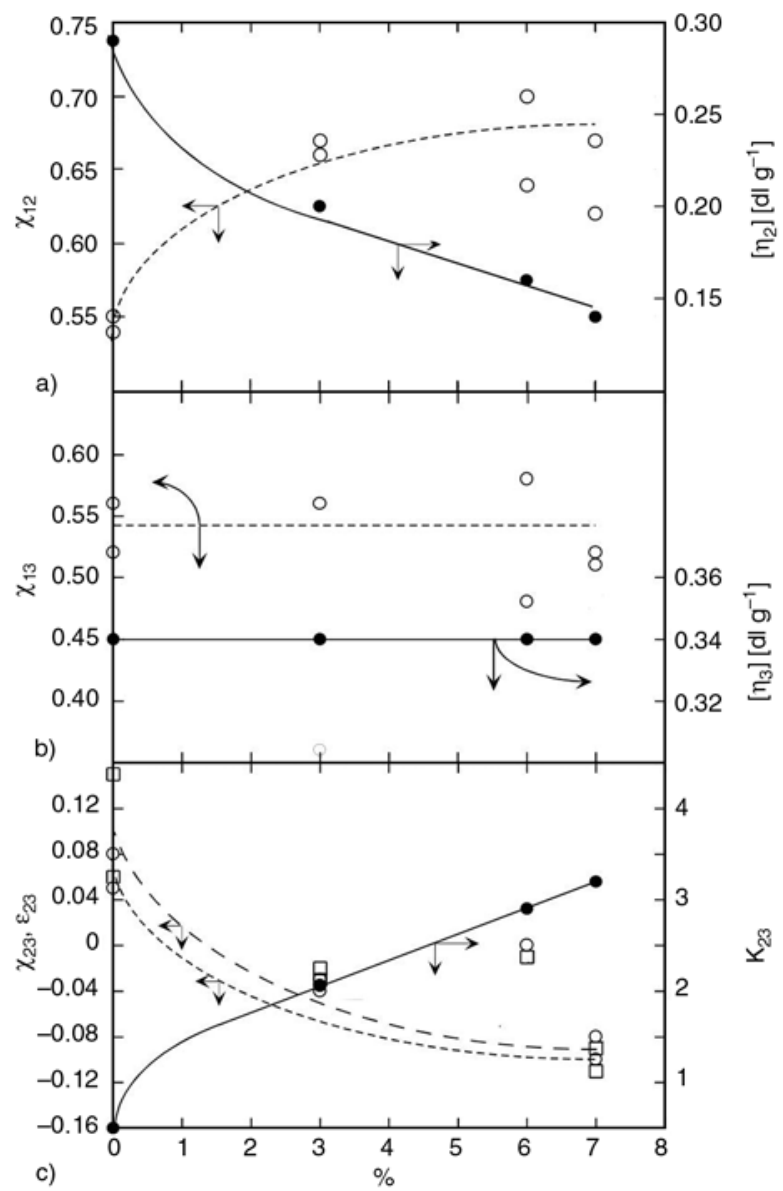

Figure 2. Variation with MAA content (in \%) of different magnitudes for the $\mathrm{CHCl}_{3}(1)$ /PSMAA-x(2)/ $\operatorname{PVPy}(3)$ systems: (a) mean interaction parameters $\bar{\chi}_{12}$ from Table $3(0,---)$ and $\left[\eta_{2}\right](\bullet,-)$; (b) mean interaction parameters $\bar{\chi}_{13}$ from Table $3(\mathrm{o},----)$ and $\left[\eta_{3}\right](\bullet,-$,$) ; and (c) \bar{\chi}_{23}$ $(\mathrm{o},----), \varepsilon_{23}(\square,-)$ and $K_{23}$ viscometric parameter $(\bullet,-)$ from [17] 
practically do not change with MAA content and are smaller than the $\chi_{12}$ ones, probably due to the weak hydrogen bonds that can be established between $\mathrm{CHCl}_{3}$ and PVPy because of the slight acid character of $\mathrm{CHCl}_{3}$ [31]. Dependences with MAA content of $\chi_{13}^{\alpha}, \chi_{13}^{\beta}$ and $\left[\eta_{3}\right]$ are shown in Figure $2 \mathrm{~b}$. As it can be seen, $\chi_{13}$ values range between 0.48 and 0.58 and $\left[\eta_{3}\right]$ values do not change with component 2 nature, which seems to indicate that this component has no influence on the 1-3 interactions. Finally, in Figure $2 \mathrm{c}$ strong $\chi_{23}$ or $\varepsilon_{23}$ dependences on copolymer PSMAA composition are observed. The calculated values even reach negative figures, typical of stable blends [14, 27], as the MAA content is raised, probably due to hydrogen bond specific interactions between MAA (H-donor) and PVPy (H-acceptor). Moreover, for these systems, the $\chi_{23}$ values can be evaluated through $\chi_{23}=\chi_{\mathrm{PS}-\mathrm{PVPy}} \phi_{\mathrm{PS}}+\chi_{\mathrm{MAA}-\mathrm{PVPy}} \phi_{\mathrm{MAA}}$ with $\phi_{\mathrm{PS}}$ and $\phi_{\text {MAA }}$ standing for the respective volume fractions of PS and MAA in the copolymer [32]. As $\chi_{\text {PS-PVPy }}$ displays positive values and decreases with rising MAA content in the copolymer, the term $\chi_{\mathrm{PS}-\mathrm{PVPy}} \phi_{\mathrm{PS}}$ will also decrease. On the other hand, $\chi_{\text {MAA-PVPy }}$ will have negative values, decreasing with rising MMA content as it does the term $\chi_{\text {MAA-PVPy }} \phi_{\text {MAA }}$. Since both terms work in the same direction, the $\chi_{23}$ values will decrease with rising MAA content in the copolymer, as seen in Figure $2 \mathrm{c}$ and in Table 3. The parallel increase of the $K_{23}$ viscometric parameter [17], also plotted in Figure $2 \mathrm{c}$, confirms the increasing stability of the polymer mixtures as the MMA content goes up. Finally, $\varepsilon_{23}$ values in these systems are small and positive in agreement with recently published ones [19].

Table 3 also gathers values of the interaction parameters calculated for $\mathrm{CHCl}_{3} / \mathrm{PSVPh}-\mathrm{y} / \mathrm{PVPy}$ systems at three different VPh contents. $\chi_{12}^{\alpha}$ and $\chi_{12}^{\beta}$ values smoothly increase with the VPh content, except for the PSVPh-7 where they slightly decrease. In any case, the increase in $\chi_{12}$ is in agreement with the decreasing $\left[\eta_{2}\right]$, as shown in Figure $3 a$. Figure $3 b$ depicts the $\chi_{13}^{\alpha}$ and $\chi_{13}^{\beta}$ dependences with VPh content and shows that $\chi_{13}$ values remain practically constant (between 0.48 and 0.60 ), as in the preceding analyzed systems. Again, it seems that the component 2 nature plays scarce if any influence on 1-3 interactions. Finally, Figure $3 \mathrm{c}$ shows the $\chi_{23}$ or $\varepsilon_{23}$ dependences on copolymer composition. As can be seen, decreasing $\chi_{23}$ or $\varepsilon_{23}$ and increasing $K_{23}$ values when rising the VPh content are the normal behaviours. However, the viscosity values and the ternary phase diagram (figure 2 in ref. [17]) were anomalous in the system $\mathrm{CHCl}_{3} / \mathrm{PSVPh}-7 / \mathrm{PVPy}$, in which a diminution of stability (stable zones outside the binodal) accounts probably due to self-associations competing with inter-associations. The anomalous $K_{23}$ decrease in the PSVPh-7 is also reflected in $\chi_{23}$ or $\varepsilon_{23}$ values here calculated, as corroborated in Figure 3c.

Similar results have been attained for systems including the $\mathrm{H}$-acceptor $\mathrm{PVP}$ as component 3 , namely: $\mathrm{CHCl}_{3} / \mathrm{PS} / \mathrm{PVP}, \mathrm{CHCl}_{3} / \mathrm{PSMAA}-3 / \mathrm{PVP}$ and $\mathrm{CHCl}_{3} / \mathrm{PSVPh}-2 / \mathrm{PVP}$, as it can be seen in Table 3. The $\chi_{12}^{\alpha}$ and $\chi_{12}^{\beta}$ and values slightly increase when rising the $\mathrm{H}$-donor comonomer content in both copolymers, in correspondence with the expected decrease of $\left[\eta_{2}\right]$ [17], as shown in Figure 4. Similar evolutions with MMA or VPh content to the previously discussed systems are also followed by the $\chi_{12}^{\alpha}, \chi_{23}^{\alpha}, \varepsilon_{12}^{\alpha}, \varepsilon_{23}^{\alpha}$, parameters. These magnitudes decrease up to small negative values with increasing $\mathrm{H}$-donor comonomer content, whereas the $K_{23}$ viscometric parameters increase, as also seen in Figure 4. As a summary, the results in Table 2, obtained through the b1

Table 3. Interaction parameters mean values from Table 2 data

\begin{tabular}{|c|c|c|c|c|c|c|c|c|}
\hline System & $\bar{\chi}_{12}^{\alpha}$ & $\bar{\chi}_{12}^{\beta}$ & $\bar{\chi}_{13}^{\alpha}$ & $\bar{\chi}_{13}^{\beta}$ & $\bar{\chi}_{23}^{\alpha}$ & $\bar{\chi}_{23}^{\beta}$ & $\bar{\varepsilon}_{23}^{\alpha}$ & $\bar{\varepsilon}_{23}^{\beta}$ \\
\hline $\mathrm{CHCl}_{3} / \mathrm{PS} / \mathrm{PVPy}$ & 0.54 & 0.55 & 0.52 & 0.56 & 0.05 & 0.08 & 0.06 & 0.15 \\
\hline $\mathrm{CHCl}_{3} / \mathrm{PSMMA-3/PVPy}$ & 0.66 & 0.67 & 0.36 & 0.56 & -0.04 & -0.03 & -0.03 & -0.02 \\
\hline $\mathrm{CHCl}_{3} / \mathrm{PSMMA-6/PVPy}$ & 0.64 & 0.70 & 0.48 & 0.58 & 0.00 & 0.00 & -0.01 & -0.01 \\
\hline $\mathrm{CHCl}_{3} / \mathrm{PSMMA-7/PVPy}$ & 0.62 & 0.67 & 0.51 & 0.52 & -0.08 & -0.10 & -0.09 & -0.11 \\
\hline $\mathrm{CHCl}_{3} / \mathrm{PSVPh}-2 / \mathrm{PVPy}$ & 0.60 & 0.62 & 0.54 & 0.60 & 0.03 & 0.00 & -0.03 & -0.03 \\
\hline $\mathrm{CHCl}_{3} / \mathrm{PSVPh}-4 / \mathrm{PVPy}$ & 0.76 & 0.76 & 0.51 & 0.60 & -0.13 & -0.23 & -0.52 & -0.68 \\
\hline $\mathrm{CHCl}_{3} / \mathrm{PSVPh}-7 / \mathrm{PVPy}$ & 0.58 & 0.59 & 0.48 & 0.54 & -0.14 & -0.14 & -0.15 & -0.15 \\
\hline $\mathrm{CHCl}_{3} / \mathrm{PS} / \mathrm{PVP}$ & 0.62 & 0.68 & 0.57 & 0.56 & 0.32 & 0.29 & 0.30 & 0.24 \\
\hline $\mathrm{CHCl}_{3} / \mathrm{PSMMA-3/PVP}$ & 0.72 & 0.71 & 0.46 & 0.52 & -0.09 & -0.08 & -0.07 & -0.01 \\
\hline $\mathrm{CHCl}_{3} / \mathrm{PSVPh}-2 / \mathrm{PVP}$ & 0.74 & 0.77 & 0.41 & 0.45 & -0.01 & -0.01 & 0.00 & 0.07 \\
\hline
\end{tabular}




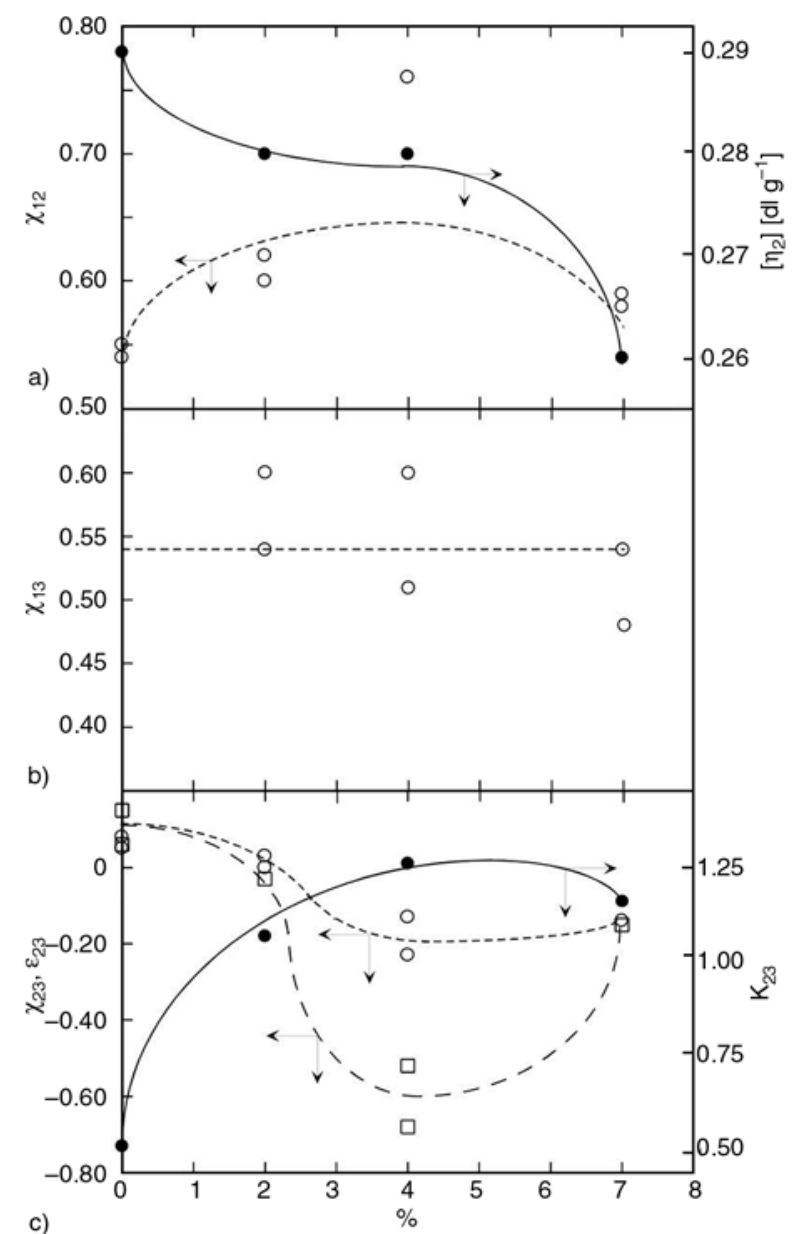

Figure 3. Variation with MAA content (in \%) of different magnitudes for the $\mathrm{CHCl}_{3}(1) / \mathrm{PSVPh}-\mathrm{y}(2) /$ PVPy(3) systems: (a) mean interaction parameters $\bar{\chi}_{12}$ from Table 3 and $\left[\eta_{2}\right]$; (b) mean interaction parameters $\bar{\chi}_{13}$ from Table 3 ; and (c) $\bar{\chi}_{23}, \varepsilon_{23}$ and $K_{23}$ viscometric parameter from [17]. Symbols as in Figure 2

model (Equations (39)-(41)) seem to be satisfactory and even values as $g_{13}=0.46_{3}$ for the $\mathrm{CHCl}_{3} / \mathrm{PVPy}$ system [24] or $g_{12}=0.54_{3}, 0.72_{0}$ for the $\mathrm{CHCl}_{3} / \mathrm{PS}$ system [24] are fairly reflected in the corresponding data of Table 2. In conclusion, it can be quoted that the b1 approach is a good enough procedure to describe and calculate the interaction parameters.

On the other hand, the molar volumes, $V_{i}$, necessary to apply the b2 model (Equations (47)-(49)) were deduced from data on specific volumes and molar masses of the components and were previously reported [17]. The application of model $\mathbf{b} 2$ leads to 6 unknowns ( $D_{i j}$ and $c_{i j}$ with $i j=12,13,23$ ) and therefore, 6 equations are necessary to deduce them: 3 of them are generated with the original compositions $\phi_{i}^{\gamma}$ and the other three by applying

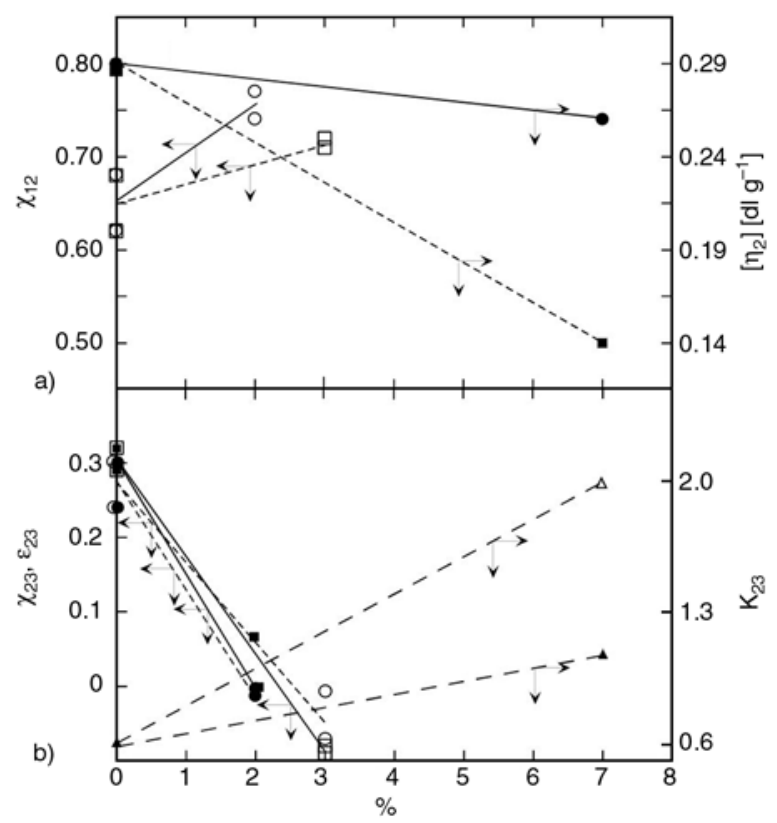

Figure 4. (a) Variation with component 2 nature and composition of $\chi_{12}$ interaction parameters for $\mathrm{CHCl}_{3}(1) / \mathrm{PSMAA}-\mathrm{x}(2) / \mathrm{PVP}(3)(\square,----)$ and $\mathrm{CHCl}_{3}(1) / \mathrm{PSVPh}-\mathrm{y}(2) / \mathrm{PVP}(3)$ systems (o, ----), as well as $\left[\eta_{2}\right]$ intrinsic viscosities from [17] for $\mathrm{CHCl}_{3}(1) / \mathrm{PSMAA}-\mathrm{x}(2) / \mathrm{PVP}(3)(\mathbf{\square},----)$ and $\mathrm{CHCl}_{3}(1) / \mathrm{PSVPh}-\mathrm{y}(2) / \mathrm{PVP}(3)$ systems $(\bullet,-)$;

(b) Variation with component 2 composition and nature of $\chi_{23}$ (squares, - ) and $\varepsilon_{23}$ (circles, ----) interaction parameters and $K_{23}$ viscometric parameters from [17] (triangles, ----) for $\mathrm{CHCl}_{3}$ (1)/PSMAA-x(2)/PVP(3) (empty symbols) and $\mathrm{CHCl}_{3}(1) / \mathrm{PSVPh}-\mathrm{y}(2) / \mathrm{PVP}(3)$ (filled symbols) systems

randomly generated compositions $u_{i}^{\gamma}$. In Table 4 are gathered $\phi_{1}^{\alpha}$ (the remaining $\phi_{i}^{\gamma}$ values have been already given in Table 1) and $u_{i}^{\gamma}$ values. Once solved the 6 equations system, it is possible to obtain the 14 interaction parameters for each composition set of any system, which are compiled in Table 5. As before, mean values of $\chi_{12}^{\gamma}, \chi_{13}^{\gamma}, \chi_{23}^{\gamma}$, and $\varepsilon_{23}^{\gamma},(\gamma=\alpha, \beta)$ have been gathered in Table 6 . For the system $\mathrm{CHCl}_{3} / \mathrm{PS} / \mathrm{PVPy}$ the $\chi_{12}^{\alpha}$ mean values seem to be acceptable, but the $\chi_{12}^{\beta}$ ones are extremely low and incomprehensible. Similar behaviours follow the $\chi_{13}^{\alpha}$ and $\chi_{13}^{\beta}$ values, that is, very low values becoming strongly negative as the $\mathrm{H}$-donor comonomer content raises. The $\chi_{13}$ small positive values for the systems $\mathrm{CHCl}_{3} / \mathrm{PVPy}$ or $\mathrm{CHCl}_{3} / \mathrm{PVP}$ are not supported by literature results, and the strongly negative ones neither can be explained by the weakly $\mathrm{H}$-donor character of $\mathrm{CHCl}_{3}$ [31]. The obtained values seem to be the 
Table 4. Experimental compositions in equilibrium, $\phi_{1}^{\alpha}$, from the binodal curve and randomly generated, $u_{i}^{\gamma}(i=1,2,3$; $\gamma=\alpha, \beta)$, through b2 model (Equations (47)-(49)) for different ternary polymer systems

\begin{tabular}{|c|c|c|c|c|c|c|}
\hline$\phi_{1}^{\alpha}$ & $\mathbf{u}_{1}^{\alpha}$ & $\mathbf{u}_{2}^{\alpha}$ & $\mathbf{u}_{3}^{\alpha}$ & $\mathbf{u}_{1}^{\beta}$ & $\mathbf{u}_{2}^{\beta}$ & $\mathbf{u}_{3}^{\beta}$ \\
\hline \multicolumn{7}{|c|}{$\mathrm{CHCl}_{3} / \mathrm{PS} / \mathrm{PVPy}$} \\
\hline 0.9607 & 0.9611 & 0.0297 & 0.0092 & 0.9720 & 0.0042 & 0.0238 \\
\hline 0.9678 & 0.9682 & 0.0194 & 0.0124 & 0.9728 & 0.0074 & 0.0198 \\
\hline 0.9559 & 0.9563 & 0.0378 & 0.0059 & 0.9748 & 0.0013 & 0.0239 \\
\hline \multicolumn{7}{|c|}{$\mathrm{CHCl}_{3} / \mathrm{PSMMA-3/PVPy}$} \\
\hline 0.7993 & 0.7996 & 0.1746 & 0.0258 & 0.9491 & 0.0072 & 0.0437 \\
\hline 0.8552 & 0.8556 & 0.1182 & 0.0262 & 0.9568 & 0.0096 & 0.0337 \\
\hline 0.9290 & 0.9294 & 0.0486 & 0.0220 & 0.9546 & 0.0180 & 0.0274 \\
\hline \multicolumn{7}{|c|}{$\mathrm{CHCl}_{3} / \mathrm{PSMMA}-6 / \mathrm{PVPy}$} \\
\hline 0.7917 & 0.7920 & 0.1735 & 0.0345 & 0.9081 & 0.0203 & 0.0716 \\
\hline 0.8240 & 0.8243 & 0.1410 & 0.0347 & 0.9047 & 0.0316 & 0.0637 \\
\hline 0.8803 & 0.8807 & 0.0723 & 0.0470 & 0.8952 & 0.0480 & 0.0568 \\
\hline \multicolumn{7}{|c|}{$\mathrm{CHCl}_{3} / \mathrm{PSMMA-7/PVPy}$} \\
\hline 0.7670 & 0.7673 & 0.2088 & 0.0239 & 0.9016 & 0.0097 & 0.0887 \\
\hline 0.868 & 0.8684 & 0.0925 & 0.0391 & 0.8965 & 0.0370 & 0.0666 \\
\hline \multicolumn{7}{|c|}{$\mathrm{CHCl}_{3} / \mathrm{PSVPh}-2 / \mathrm{PVPy}$} \\
\hline 0.9009 & 0.9013 & 0.0843 & 0.0144 & 0.9610 & 0.0080 & 0.0310 \\
\hline 0.9369 & 0.9373 & 0.0463 & 0.0164 & 0.9623 & 0.0109 & 0.0268 \\
\hline 0.9541 & 0.9545 & 0.0241 & 0.0214 & 0.9611 & 0.0134 & 0.0255 \\
\hline \multicolumn{7}{|c|}{$\mathrm{CHCl}_{3} / \mathrm{PSVPh}-4 / \mathrm{PVPy}$} \\
\hline 0.8833 & 0.8837 & 0.0814 & 0.0349 & 0.9411 & 0.0558 & 0.0533 \\
\hline 0.9238 & 0.9242 & 0.0445 & 0.0313 & 0.9410 & 0.0160 & 0.0429 \\
\hline \multicolumn{7}{|c|}{$\mathrm{CHCl}_{3} / \mathrm{PSVPh}-7 / \mathrm{PVPy}$} \\
\hline 0.9211 & 0.9215 & 0.0646 & 0.0139 & 0.9638 & 0.0051 & 0.0311 \\
\hline 0.9397 & 0.9410 & 0.0446 & 0.0153 & 0.9596 & 0.0138 & 0.0266 \\
\hline \multicolumn{7}{|c|}{$\mathrm{CHCl}_{3} / \mathrm{PS} / \mathrm{PVP}$} \\
\hline 0.9212 & 0.9216 & 0.0675 & 0.0109 & 0.9479 & 0.0154 & 0.0367 \\
\hline 0.9349 & 0.9353 & 0.0498 & 0.0149 & 0.9490 & 0.0188 & 0.0322 \\
\hline \multicolumn{7}{|c|}{$\mathrm{CHCl}_{3} / \mathrm{PSMMA-3/PVP}$} \\
\hline 0.8277 & 0.8280 & 0.0891 & 0.0828 & 0.8960 & 0.0024 & 0.1016 \\
\hline 0.8741 & 0.8718 & 0.0467 & 0.0815 & 0.8874 & 0.0207 & 0.0919 \\
\hline \multicolumn{7}{|c|}{$\mathrm{CHCl}_{3} / \mathrm{PSVPh}-2 / \mathrm{PVP}$} \\
\hline 0.8370 & 0.8373 & 0.0927 & 0.0699 & 0.8986 & 0.0067 & 0.0948 \\
\hline 0.8781 & 0.8785 & 0.0447 & 0.0768 & 0.8955 & 0.0168 & 0.0877 \\
\hline
\end{tabular}

most appropriate, since these magnitudes use to display small positive [19] or negative [14, 26] values in blends. However, as seen before and as expected, they do not become more negative with increasing the H-donor content of copolymer neither follow the expected viscometric behaviour [17]. As a summary, obtained results from b2 model (Equations (47)-(49)) are unsatisfactory, in sharp contrast with those determined with the b1 model (Equations (39)-(41)). Probably, the main reason for these results is the fact that the $\alpha$ parameter is used in the $\mathbf{b} \mathbf{1}$ model but ignored in the $\mathbf{b} \mathbf{2}$ approach. As a conclusion, the obtained interaction parameters through the b1 approach are considerably better than those coming from $\mathbf{b} \mathbf{2}$ approach, in spite of the need of an additional fitting parameter in the former, an aspect long supported for binary solvent/polymer systems [33].

\section{Conclusions}

Two theoretical approaches to calculate $\chi_{i j}$ or $\varepsilon_{i j}$ and $g_{i j}$ interaction parameters in ternary polymer systems with specific interactions have been proposed. The validity of both models has been checked and assessed by comparison between the calculated values and those experimentally obtained through ternary phase diagrams. In general, the values calculated with model b1 (Equations (39)-(41)) show a fair agreement with the experimental ones found in the literature. Moreover, in the systems where the component 2 is a 
Table 5. Interaction parameters evaluated from Equations (47)-(49) for different ternary polymer systems

\begin{tabular}{|c|c|c|c|c|c|c|c|c|c|c|c|c|c|c|}
\hline$\phi_{1}^{\alpha}$ & $\chi_{12}^{\alpha}$ & $\chi_{12}^{\beta}$ & $\chi_{13}^{\alpha}$ & $\chi_{13}^{\beta}$ & $\chi_{23}^{\alpha}$ & $\chi_{23}^{\beta}$ & $\varepsilon_{23}^{\alpha}$ & $\varepsilon_{23}^{\beta}$ & $\mathbf{g}_{12}^{\alpha}$ & $\mathbf{g}_{12}^{\beta}$ & $\mathbf{g}_{13}^{\alpha}$ & $\mathbf{g}_{13}^{\beta}$ & $\mathbf{g}_{23}^{\alpha}$ & $\mathbf{g}_{23}^{\beta}$ \\
\hline \multicolumn{15}{|c|}{$\mathrm{CHCl}_{3} / \mathrm{PS} / \mathrm{PVPy}$} \\
\hline 0.9607 & 5066 & 0.0727 & 0.1146 & 0.3439 & 0.0247 & 0.0249 & 0.0022 & 0.0035 & 0.0160 & 0.0018 & 0.0050 & 0.0101 & 0.6549 & 1.1110 \\
\hline 0.9678 & 405 & 0.2228 & .0466 & .0637 & 0.0452 & 0.0056 & 0006 & 0008 & 0141 & .0053 & .0489 & 0.0504 & 1614 & 2074 \\
\hline 559 & 485 & .0297 & .0291 & 0.0834 & 0211 & 0.0254 & 0.0015 & 0.0037 & 0209 & 0.0070 & 0.0021 & .0040 & 4158 & 1.0347 \\
\hline \multicolumn{15}{|c|}{$\mathrm{CHCl}_{3} / \mathrm{PSMMA-3/PVPy}$} \\
\hline 7993 & 489 & 523 & 08 & .3319 & 19 & -0.0282 & 0.0005 & 0.0007 & 1124 & 0032 & 8730 & 0.9370 & 1301 & 1944 \\
\hline 8552 & 5388 & 0.0889 & .2358 & 0.9588 & 0.1793 & 0.2316 & 0007 & 0.0008 & 0665 & .0037 & 1.1279 & 1.1377 & 1712 & .1970 \\
\hline 290 & 4674 & 0.1402 & .1137 & 0.1975 & 0.0402 & -0.0408 & 0.0003 & 0.0003 & .0207 & .0054 & 0.2416 & 0.2426 & 0.0750 & 0.0810 \\
\hline \multicolumn{15}{|c|}{$\mathrm{CHCl}_{3} / \mathrm{PSMMA-6/PVPy}$} \\
\hline 7917 & 5747 & 0.1232 & .0364 & .1013 & 83 & 0.2574 & 0.0001 & 0.0002 & 1410 & .0127 & 2740 & 2904 & 0289 & .0592 \\
\hline 8240 & 5888 & 0.0755 & .1061 & .2283 & 0.2525 & 0.4653 & .0002 & 0.0003 & .0592 & 0.0071 & 0.6076 & 0.6366 & .0450 & 0.0795 \\
\hline 8803 & 2396 & 0.0053 & 0.5110 & 0.6165 & 0.0978 & -0.0971 & 0.0019 & 0.0022 & 0.0015 & .0003 & 1.0170 & 1.0323 & .5283 & 0.5881 \\
\hline \multicolumn{15}{|c|}{$\mathrm{CHCl}_{3} / \mathrm{PSMMA-7/PVPy}$} \\
\hline 670 & 175 & 0.0680 & .0431 & .1317 & 0.0307 & 0.1838 & 0.0003 & 0.0011 & 0.1346 & .0051 & 0.3673 & 0.4094 & .0630 & 0.2482 \\
\hline 8680 & 3650 & 0.0359 & 0.3033 & .2969 & 0.0549 & 0.0626 & 0.0033 & 0.0101 & 0.1650 & .0032 & 0.0342 & 0.0741 & 0.6810 & 1.5535 \\
\hline \multicolumn{15}{|c|}{$\mathrm{CHCl}_{3} / \mathrm{PSVPh}-2 / \mathrm{PVPy}$} \\
\hline 9009 & 118 & 0.0591 & .1178 & .3544 & 0.1823 & 0.3546 & 0.0001 & 0.0002 & 0.0324 & .0021 & 0.3460 & 0.3590 & 0.0390 & 0.0746 \\
\hline 0.9369 & 3796 & 0.0249 & .1983 & 0.3770 & 0.1993 & 0.2840 & 0.0001 & 0.0002 & .0074 & .0008 & 0.3552 & 0.3621 & .0526 & 0.0741 \\
\hline 9541 & 1806 & 0.0182 & .1404 & .1830 & 0.1018 & 0.1023 & 0.0003 & 0.0003 & 0.0017 & 0.0005 & 0.1773 & 0.1774 & 0.1267 & 0.1240 \\
\hline \multicolumn{15}{|c|}{$\mathrm{CHCl}_{3} / \mathrm{PSVPh}-4 / \mathrm{PVPy}$} \\
\hline 833 & 600 & -0.0683 & .5441 & .9430 & .0001 & -0.0001 & 0.0000 & 0.0000 & 0524 & 0034 & 0.0670 & 0.1006 & .0176 & 0.0181 \\
\hline 9238 & 175 & 0.0056 & 3700 & .5203 & 0.0053 & -0.0014 & 0.0005 & 0.0008 & .0025 & 0003 & 0.5899 & 0.6017 & 0.1280 & 0.1967 \\
\hline \multicolumn{15}{|c|}{$\mathrm{CHCl}_{3} / \mathrm{PSVPh}-7 / \mathrm{PVPy}$} \\
\hline 0.9211 & 5636 & 0.1174 & .0030 & 0.0076 & 0.0039 & 0.0064 & 0.0000 & 0.0000 & 0.0410 & 0.0030 & 0.0076 & 0.0079 & 0.0109 & 0.0210 \\
\hline 0.9397 & 5512 & 0.2829 & .0206 & 0.0346 & 0.0225 & 0.0338 & 0.0000 & 0.0000 & 0.0342 & 0.0103 & 0.0384 & 0.0392 & 0.0004 & 0.0006 \\
\hline \multicolumn{15}{|c|}{$\mathrm{CHCl}_{3} / \mathrm{PS} / \mathrm{PVP}$} \\
\hline 9212 & 279 & 0.0270 & 201 & 0.1283 & 0.0527 & 0.0647 & 0.0022 & 0.0045 & 0.0117 & 0.0012 & 0.0016 & 0.0055 & .6125 & 1.3915 \\
\hline 0.9349 & 3065 & 0.0748 & 0.0421 & 0.0592 & 0.0234 & 0.0246 & 0.0013 & 0.0019 & 0.0123 & 0.0032 & 0.0782 & 0.0811 & 0.3676 & 0.5789 \\
\hline \multicolumn{15}{|c|}{$\mathrm{CHCl}_{3} / \mathrm{PSMMA-3/PVP}$} \\
\hline 0.8277 & 4470 & 0.0055 & 0.8054 & 1.5043 & -0.0680 & -0.0633 & 0.0008 & 0.0009 & .0451 & 0.0006 & 0.8576 & 0.9010 & 0.2037 & 0.2388 \\
\hline 0.8714 & 2230 & 0.0042 & .2872 & 0.3460 & -0.0414 & -0.0415 & 0.0006 & 0.0006 & 0.0026 & .0004 & 0.7975 & .8069 & 1536 & 0.1655 \\
\hline \multicolumn{15}{|c|}{$\mathrm{CHCl}_{3} / \mathrm{PSVPh}-2 / \mathrm{PVP}$} \\
\hline 0.8370 & 0.4245 & 0.0515 & -0.0503 & -0.0911 & 0.1362 & 0.1861 & 0.0000 & 0.0001 & 0.0496 & 0.0033 & 0.2941 & 0.3037 & 0.0126 & 0.0168 \\
\hline 0.8781 & 0.2576 & 0.0033 & -0.6400 & 0.7908 & 0.2147 & 0.2542 & 0.0013 & 0.0014 & 0.0026 & 0.0003 & 0.9441 & 0.9600 & 0.4402 & 0.4900 \\
\hline
\end{tabular}

Table 6. Interaction parameters mean values from Table 5 data

\begin{tabular}{|l|c|c|c|c|c|c|c|c|}
\hline \multicolumn{1}{|c|}{ System } & $\bar{\chi}_{\mathbf{1 2}}^{\alpha}$ & $\bar{\chi}_{\mathbf{1 2}}^{\beta}$ & $\bar{\chi}_{\mathbf{1 3}}^{\alpha}$ & $\bar{\chi}_{\mathbf{1 3}}^{\beta}$ & $\bar{\chi}_{\mathbf{2 3}}^{\alpha}$ & $\bar{\chi}_{\mathbf{2 3}}^{\beta}$ & $\bar{\varepsilon}_{\mathbf{2 3}}^{\alpha}$ & $\overline{\boldsymbol{\varepsilon}}_{\mathbf{2 3}}^{\beta}$ \\
\hline $\mathrm{CHCl}_{3} / \mathrm{PS} / \mathrm{PVPy}$ & 0.47 & 0.11 & 0.03 & 0.12 & 0.03 & 0.04 & 0.00 & 0.00 \\
\hline $\mathrm{CHCl}_{3} / \mathrm{PSMMA-3/PVPy}$ & 0.52 & 0.10 & -0.27 & $-1,16$ & 0.03 & 0.05 & 0.00 & 0.00 \\
$\mathrm{CHCl}_{3} / \mathrm{PSMMA-6/PVPy}$ & 0.47 & 0.07 & -0.22 & -0.32 & 0.09 & 0.21 & 0.00 & 0.00 \\
$\mathrm{CHCl}_{3} / \mathrm{PSMMA-7/PVPy}$ & 0.44 & 0.05 & -0.17 & -0.71 & 0.04 & 0.12 & 0.00 & 0.01 \\
\hline $\mathrm{CHCl}_{3} / \mathrm{PSVPh}-2 / \mathrm{PVPy}$ & 0.32 & 0.03 & -0.15 & -0.30 & 0.16 & 0.25 & 0.00 & 0.00 \\
$\mathrm{CHCl}_{3} / \mathrm{PSVPh}-4 / \mathrm{PVPy}$ & 0.44 & 0.04 & -0.46 & -1.23 & 0.00 & 0.00 & 0.00 & 0.00 \\
$\mathrm{CHCl}_{3} / \mathrm{PSVPh}-7 / \mathrm{PVPy}$ & 0.56 & 0.20 & -0.01 & -0.02 & 0.01 & 0.02 & 0.00 & 0.00 \\
\hline $\mathrm{CHCl}_{3} / \mathrm{PS} / \mathrm{PVP}$ & 0.37 & 0.05 & -0.01 & 0.03 & 0.04 & 0.04 & 0.00 & 0.00 \\
$\mathrm{CHCl}_{3} / \mathrm{PSMMA-3/PVP}$ & 0.34 & 0.01 & -0.55 & -0.93 & -0.05 & -0.05 & 0.00 & 0.01 \\
$\mathrm{CHCl}_{3} / \mathrm{PSVPh}-2 / \mathrm{PVP}$ & 0.34 & 0.03 & -0.35 & -0.44 & 0.18 & 0.22 & 0.00 & 0.00 \\
\hline
\end{tabular}

copolymer, the $\chi_{13}$ values practically do not change with the copolymer content (MMA or VPh) denoting that the chemical nature of this component has no influence on the 1-3 interactions. On the contrary, strong $\chi_{23}$ or $\varepsilon_{23}$ dependences on copolymer composition are observed. The calculated values even reach negative figures, typical of stable blends, as the comonomer content is raised, probably due to hydrogen bond specific interactions between the H-donor (PSMAA or PSVPh) and the $\mathrm{H}$-acceptor (PVPy or PVP), in correspondence with the experimental viscometric data. However, the 
obtained results from b2 model (Equations (47)(49)) are quite unsatisfactory, in sharp contrast with those determined with the $\mathbf{b} \mathbf{1}$ model, most probably due to the fact that the later model contains an empirical entropy correction ( $\alpha$ parameter) that is ignored in the other approach. Therefore, as a main conclusion, it can be quoted that the b1 approach is a good enough mathematical procedure to describe and calculate interaction parameters, at least for the TPS here compared and for all the phase compositions assayed.

\section{Acknowledgements}

The authors acknowledge financial support from Ministerio de Educación y Ciencia, Spain, through the Project: MAT2006-03997.

\section{References}

[1] Bouslah N., Amrani F.: Miscibility and specific interactions in blends of poly[(styrene)-co-(cinnamic acid)] with poly(methyl methacrylate) and modified poly(methyl methacrylate). Express Polymer Letter, 1, 44-50 (2007).

[2] Barton A. F. M.: Handbook of solubility parameters and other cohesion parameters. CRC Press, Boca Raton (1983).

[3] Barton A. F. M.: Handbook of polymer-liquid interaction parameters and solubility parameters. CRC Press, Boca Raton (1990).

[4] Kamide K.: Thermodynamics of polymer solutions: Phase equilibria and critical phenomena. Elsevier, Amsterdam (1990).

[5] Uriarte C., Fernandez-Berridi M. J., Elorza J. M., Iruin J. J., Kleintjens L.: Determination of the interaction parameter $g$ by inverse gas chromatography: An additional experimental test of the classic lattice model. Polymer, 30, 1493-1497 (1989).

[6] Staverman A. J.: Cohesive energy of liquid mixtures I. Recueil des Travaux Chimiques, 56, 885-890 (1937).

[7] Koningsveld R., Kleintjens L. A.: Liquid-liquid phase separation in multicomponent polymer systems. X. Concentration dependence of the pair-interaction parameter in the system cyclohexane-polystyrene. Macromolecules, 4, 637-641 (1971).

[8] Bondi A.: Van der Waals volumes and radii. Journal of Physical Chemistry, 68, 441-451 (1964).

[9] Bae Y. C., Shim J. J., Soane D. S., Prausnitz J. M.: Representation of vapor-liquid and liquid-liquid equilibria for binary systems containing polymers: Applicability of an extended Flory-Huggins equation. Journal of Applied Polymer Science, 47, 1193-1206 (1993).
[10] Bae Y. C., Lambert S. M., Soane D. S., Prausnitz J. M.: Cloud-point curves of polymer solutions from thermooptical measurements. Macromolecules, 24, 4403-4407 (1991).

[11] Rico M., Ramirez C., Montero B., Diez J., Lopez J.: Phase diagram for a system of polydisperse components consisting of the precursor of an epoxy/diamine thermoset and a thermoplastic: Analysis based on a lattice theory model. Macromolecular Theory and Simulations, 15, 487-496 (2006).

[12] Riccardi C. C., Borrajo J., Meynie L., Fenouillot F., Pascault J-P.: Thermodynamic analysis of the phase separation during the polymerization of a thermoset system into a thermoplastic matrix. Part II. Prediction of the phase composition and the volume fraction of the dispersed phase. Journal of Polymer Science, Part B: Polymer Physics, 42, 1361-1368 (2004).

[13] Schreiber H. P., Patterson D., Tewari Y. B., Guillet J. E.: Application of gas-liquid chromatography to the thermodynamics of polymer solutions. Macromolecules, 4, 356-359 (1971).

[14] Uriarte C., Iruin J. J., Fernandez-Berridi M. J., Elorza J. M.: Chromatographic studies of a poly(vinyl methyl ether)/phenoxy resin blend near the lower critical solution temperature. Polymer, 30, 1155-1159 (1989).

[15] Schuld N., Wolf B. A.: Polymer-solvent interaction parameters. in 'Polymer Handbook' (eds.: Brandrup J., Immergut E. H., Grulke E. A.), Wiley-Interscience, New York, 247-264 (1999).

[16] Mumby S. J., Sher R.: Determination of $\chi$ from liquidliquid phase data and the computation of phase diagrams for quasi-binary polymer solutions and blends. Macromolecules, 27, 689-694 (1994).

[17] Torrens F., Soria V., Codoñer A., Abad C., Campos A.: Compatibility between polystyrene copolymers and polymers in solution via hydrogen bonding. European Polymer Journal, 42, 2807-2823 (2006).

[18] Soria V., Figueruelo J. E., Gómez C. M., Abad C., Campos A.: Ternary polymer solutions with hydrogen bonds. 1. Theory. Macromolecular Theory and Simulations, 16, 53-61 (2007).

[19] Figueruelo J. E., Monzó I. S., Gómez C. M., Soria V., Abad C., Campos A.: Ternary polymer solutions with hydrogen bonds. 2. Prediction of Phase Diagrams. Macromolecular Theory and Simulations, 16, 62-76 (2007).

[20] Gómez C. M., Verdejo E., Figueruelo J. E., Campos A., Soria V.: On the thermodynamic treatment of poly(vinylidene fluoride)/polystyrene blend under liquid-liquid phase separation conditions. Polymer, 36, 1487-1498 (1995).

[21] Campos A., Gómez C. M., García R., Figueruelo J. E., Soria V.: Extension of the Flory-Huggins theory to study incompatible polymer blends in solution from phase separation data. Polymer, 37, 3361-3372 (1996). 
[22] Gómez C. M., Figueruelo J. E., Campos A.: Evaluation of thermodynamic parameters for blends of polyether sulfone and poly(methyl methacrylate) or polystyrene in dimethylformamide. Polymer, 39, 4023-4032 (1998).

[23] Gómez C. M., Porcar I., Monzo I. S., Abad C., Campos A.: Modelling the influence of nanoparticles in the phase behaviour of an epoxy/polystyrene mixture. European Polymer Journal, 43, 360-373 (2007).

[24] Campos A., Gavara R., Tejero R., Gomez C., Celda B.: A Flory-Huggins thermodynamic approach for predicting sorption equilibrium in ternary polymer systems. Journal of Polymer Science, Part B: Polymer Physics, 27, 1569-1597 (1989).

[25] Torrens F., Monzó I. S., Gómez C. M., Abad C., Campos A.: Study and comparison of interaction parameter and phase behaviour of epoxy/polystyrene and epoxies copolymer polystyrene-b-polymethylmethacrylate blends. Polymer Composites (in press) DOI: 10.1002/pc.20518 (2008)

[26] Wolfram S.: The mathematica book. Wolfram Media, Champaign (2003).
[27] Prolongo M. G., Masegosa R. M., Horta A.: Polymerpolymer interaction parameter in the presence of a solvent. Macromolecules, 22, 4346-4351 (1989).

[28] Masegosa R. M., Prolongo M. G., Horta A.: The $g$ interaction parameter of polymer-solvent systems. Macromolecules, 19, 1478-1486 (1986).

[29] Figueruelo J. E., Celda B., Campos A.: Predictability of properties in ternary solvent (1)/solvent(2)/polymer(3) systems from interaction parameters of the binary systems. 1. General considerations and evaluation of preferential solvation coefficients. Macromolecules, 18, 2504-2511 (1985).

[30] Brandrup J., Immergut E. H., Grulke E. A.: Polymer Handbook, Wiley Interscience, New York (1999).

[31] Marcus Y.: The properties of solvents. John Wiley and Sons, New York (1998).

[32] Champetier G., Monnerie L.: Introduction a la chimie macromoleculaire. Masson and Cie, Paris (1969).

[33] Walsh D. J., Higgins J. S., Maconnachie A.: Polymer blends and mixtures. NATO ASI Series E: Applied Science (1985). 